\title{
1 Proteomic landscape of multi-layered breast cancer internal tumor heterogeneity
}

2 Mariya Mardamshina ${ }^{1,2}$, Anjana Shenoy ${ }^{1}$, Daniela Necula ${ }^{3}$, Kateryna Krol ${ }^{3}$, Daniel Pirak ${ }^{4}$, Nitay

3 Itzhacky 4 , Irina Marin ${ }^{3}$, Bruria Shalmon³, Roded Sharan ${ }^{4}$, Einav Gal-Yam ${ }^{5}$, Iris Barshack ${ }^{3}$, Tamar

4 Geiger $^{1,2 *}$.

$5 \quad{ }^{1}$ Department of Human Molecular Genetics and Biochemistry, Tel Aviv University, Tel Aviv, Israel;

$6 \quad{ }^{2}$ Department of Molecular Cell Biology, Weizmann Institute of Science, Rehovot, Israel;

$7 \quad{ }^{3}$ Pathology Institute, Sheba Medical Center, Tel Hashomer, Ramat Gan, Israel;

$8 \quad{ }^{4}$ School of Computer Science, Tel Aviv University, Tel Aviv, Israel;

$9{ }^{5}$ Oncology Department, Sheba Medical Center, Tel Hashomer, Ramat Gan, Israel;

$11{ }^{*}$ Correspondence: Tamar Geiger tami.geiger@weizmann.ac.il 


\section{Summary}

Despite extensive research, internal tumor heterogeneity presents enormous challenges to achieve complete therapeutic responses. Changes in protein expression are central determinants of cancer phenotypes that reflect potential therapeutic targets. However, previous proteomic studies did not address internal heterogeneity, therefore, masked the necessary spatial resolution to achieve a comprehensive understanding of cancer complexity. Here we present the first large-scale multi-focal breast cancer proteomic study of $\mathbf{3 3 0}$ tumor regions which associated cancer cell function, pathological parameters, and spatial localization of each tumor region. We found marked internal proteomic heterogeneity even within tumors presenting homogeneous receptor expression. Additionally, analysis of the internal heterogeneity, based on coexisting receptor expression or histological patterns in single tumors, showed significant functional differences between homogeneous and heterogeneous tumors related to cancer metabolism, immunogenicity, and proliferation. We anticipate that this study will serve as a starting point towards the development of improved cancer therapy and diagnostics.

\section{Main}

The interplay between cancer genetic aberrations and external microenvironmental cues is the basis of tumorigenic growth, metastasis, and development of highly heterogeneous tumors. Although tumor heterogeneity has been thoroughly investigated at the genomic and transcriptomic levels ${ }^{1-3}$, limited studies have investigated heterogeneity at the proteomic level ${ }^{4}$. Routine breast cancer classification is still based on few molecular markers (i.e., estrogen receptor, progesterone receptor, and HER2); however, limitation to the known breast cancer receptors neglects additional protein determinants of the tumors and even fails to account for the heterogeneous expression of these markers ${ }^{5-11}$. Here, we aimed to examine the proteomic landscapes within single tumors, irrespective of their clinical subtype, and investigate the importance of the heterogeneous characteristics of the tumors for their progression. 


\section{Spatially-resolved multi-region proteomics}

36 To comprehensively examine proteomic internal breast cancer heterogeneity, and associate cellular

37 functions with histopathological diversity, we performed IHC-guided multi-focal proteomic analysis of 330 histopathologically mapped regions derived from 35 treatment-naive primary breast tumors (Fig. 1A, B; Extended Data Fig. 1A; Supplementary Table 1A-B). Altogether our cohort was comprised of 311 tumor regions, including 12 non-invasive cancer regions and 19 regions from normal adjacent healthy breast tissue, as controls from 13 patients (Extended Data Fig. 1A; nine regions on average per tumor; Supplementary Table 1B). As a preliminary validation of the proteomic data, we examined subtypespecific differences, which recapitulated known characteristics, such as cytokeratin signatures, proliferation, and metabolic functions (Extended Data Fig. 1B-E). Global unsupervised clustering of all regions based on the entire proteome revealed a multilayered complexity of receptor expression patterns, histology, and patient identity. Tumor regions are segregated into four main clusters (Fig. 1C), primarily based on receptor expression (Fig. 1D). Hormone receptor-positive $(\mathrm{HR}+)$ clusters were further segregated based on the histological characteristics (Fig. 1E, F). Normal samples were clustered together irrespective of patient identity and closer to the receptor-positive regions, while tumor grade did not have a major effect on clustering (Fig. 1D, E). Interestingly, we found that TN regions that are embedded in HR+ tumors

51 are commonly clustered with their tumor of origin, while several tumors with strong HER2+ expression

52 formed a distinct cluster. including known subtype markers like HER2/ErbB2, Muc1, CD44, and keratins (Extended Data Fig. 2A, B).

56 In addition, by combining the multisampling technique with the physical coordinates of each region within 57 the tumor, we found proteomic determinants of their spatial localization. HR+ regions closer to the tumor 
potential ${ }^{12}$, while peripheral regions from TN tumors appear to be more proliferative with higher antigen presentation than their internal counterparts (Fig. 1G). Furthermore, we found the FSCN1 and SRC, which are known to be associated with cell migration, invasion, and high metastatic potential to be highly expressed in peripheral regions, irrespective of receptor expression ${ }^{13,14}$.

\section{Quantification of proteomic heterogeneity}

64

Considering the proteomic differences in spatially resolved regions within single tumors, we opt to quantitatively measure proteomics-based internal tumor heterogeneity (ITH). First, we constructed an internal variability score for each protein in each tumor using the proteomic data, which enabled the examination of the more variable and more stable processes. In agreement with the spatial tumor analysis, we found focal adhesion, keratins, and immune processes (phagocytosis and interferonmediated signaling) to be among the most variable processes (Fig. 2A). In addition, metabolic processes, including cholesterol and carbohydrate metabolism presented high intratumor regional heterogeneity. High variability of immune processes might be associated with low immunotherapy response of breast cancer in general, as most tumors harbor regions with low immune signals. In the group of homogeneously-expressed proteins, we found enriched cell cycle-related, translation-related processes, as well as proteasome and ERAD pathways (Fig. 2B). These analyses suggest that similar to targeting clonal mutations, homogeneously-expressed proteins (e.g., cell cycle-related ${ }^{15}$ or proteasome-related ${ }^{16}$ ) may serve as potential therapeutic targets in combination with subtype-specific agents. We propose that these might be critical in achieving complete therapeutic responses. In contrast, targeting highly variable proteins may be insufficient to target the entire tumors.

To complement the unsupervised variability analyses, we asked whether tumors possess homogeneous malignancy potential. Similar to the progression of disease approach, applied in genomic analyses ${ }^{17}$, we calculated a malignancy score per tumor region based on the correlation between its proteome and the average healthy control tissue (Extended Data Fig. 3A; see Methods). The malignancy 
score shows the extent of change from the healthy tissue and reflects the progression of a transformed phenotype, and was validated using grade of tumor progression (Extended Data Fig. 3B-D). Of note, while all regions within each tumor were defined as the same grade (excluding DCIS), most tumors showed a marked variance of malignancy scores of the different regions (Fig. 2C). Next, similar to genomic ITH scores, we calculated a proteomic heterogeneity score (P-ITH) per tumor as the average of two nonoverlapping pairs of regions with the lowest correlation (Extended Data Fig. 3A; see Methods). To examine the interplay between the proteomics-based scores, we plotted the distribution of regional scores within each tumor and color-coded each tumor based on its P-ITH score (Fig. 2C). Tumors with highly variable proteomic features showed association with more diverging malignancy scores, which reflects the functional importance of the tumor proteomic heterogeneity.

\section{Molecular and histological subtype heterogeneity}

Our histopathological tumor maps showed high variability of molecular subtypes (based on receptor expression) and histological subtypes. Sixty-six percent of the tumors in our cohort presented heterogeneous receptor expression levels that included $H R+(E R+/ P R+/ E R P R+)$ and TN regions (Extended Data Fig. 3E). Examination of the co-existence of distinct histological subtypes within single tumors showed that $17 \%$ of the tumors included more than one subtype (Extended Data Fig. 3F). Aiming to associate between the proteomic heterogeneity and the molecular and histological heterogeneity, we constructed additional heterogeneity scores based on pathological parameters: A molecular subtype heterogeneity score (M-ITH) and a histological subtype heterogeneity score $(\mathrm{H}-\mathrm{ITH})$. M-ITH and H-ITH scores were constructed similar to the Shannon diversity index ${ }^{18}$; however, we modified the formula to include the fraction of tumor area belonging to one subtype, and the number of coexisting subtypes within each tumor (Extended Data Fig. 3G; see Methods). Higher ITH scores correspond to a larger number of different subtypes within a single tumor and a lower dominance of a single subtype. We then examined the impact of the $\mathrm{M}$ - and $\mathrm{H}$-ITH scores on the proteome. Canonical correlation analysis determined the 

components, which explain the largest fraction of the variance, showed the highest correlation with $\mathrm{H}$ -

Extended Data Fig. 4A). These results show the importance of the histopathologically heterogeneous nature of the tumors in shaping its proteome. cancer progression.

\section{Functional landscapes of tumor heterogeneity}

123 Given the significance of the internal tumor heterogeneity, we aimed to understand the functions

124 associated with each of the heterogeneity scores. We extracted all proteins that correlated with each one

127 String database (Fig. 3A). Separate networks, colored for each score, reflected the higher levels of proliferation-related proteins and protein translation and turnover in the high malignancy scores. A mirror 
presentation and cell cycle were enriched in high $\mathrm{H}-\mathrm{ITH}$; high IFNg and antigen presentation related proteins were enriched in high P-ITH scores (Extended Data Fig. 4C, D). molecular subtype separately (Fig. 3B). In accordance with the previous results, we found that homogeneous ERPR+, HER2+, and TN regions correlate with Grade 3, while when these molecular subtypes originated from heterogeneous tumors, they mostly correlated with Grade 2 (Extended Data Fig.

$1374 \mathrm{E}$; see Methods). Therefore, the molecular heterogeneity is reduced with a higher grade, irrespective of the molecular subtype. In contrast, examination of the histological subtypes showed that homogeneous MPC, PLM, and ILC significantly correlated with Grade 2, while IDC-NST and MPC regions from heterogeneous tumors positively correlated with Grade 3 (Extended Data Fig. 4E). These results suggest that the histological subtypes (and specifically the combination of MPC and IDC-NST) diverge and are not selected against during breast cancer progression.

To associate between the heterogeneity status and all available clinical parameters and protein expression patterns, we performed unsupervised weighted gene co-expression network analysis

145 (WGCNA; Extended Data Fig. 5). First, we observed a clear separation of the module correlation based on tumor grade (Fig. 4A; Extended Data Fig. 6A); modules with a strong positive correlation with Grade2

147 demonstrated a negative correlation with Grade 3, and vice-versa. In agreement with our previous results, 148 the M-ITH score and Grade 2 mostly correlated with the same modules, which were enriched for 149 mitochondrial metabolism, cell adhesion, and innate immunity (Fig. 4A). A closer look at the most highly correlating of these modules, MEturquoise, showed a high positive correlation with heterogeneous

151 tumors of all subtypes, and negative correlations with high grade and TN-homogeneous tumors (Fig. 4B).

152 In contrast to Grade2, in Grade 3 where we saw the increased molecular subtype homogeneity, none of 153 the modules were common to both $\mathrm{HR}+$ homogeneous and $\mathrm{TN}$ homogeneous regions, suggesting that 154 distinct processes dominate tumor progression in a molecular subtype-specific manner. 


\section{Impact of immune processes on tumor heterogeneity}

156 Enrichment analysis of the WGCNA modules showed three modules, MElightgreen, MEmagenta, and

157 MEpurple, enriched for immune processes. The MElightgreen module, which was enriched for IFN

158 signaling, highly correlated with Grade 3, and homogeneous ERPR regions (Fig. 4C; Extended Data Fig. 6B),

159 suggesting the involvement of anti-tumor immunity in eliciting high-grade homogeneity in this subtype.

160 MEmagenta highly correlated with homogeneous TN tumors, heterogeneous IDC-NST, Grade 3 and high

161 P-ITH, and negatively correlated with Grade 2 (Fig. 4D; Extended Data Fig. 6B). This module was enriched

162 for antigen processing and presentation, and included multiple HLA proteins and TAP proteins, suggesting

163 higher visibility to the immune system in high-grade TN tumors with coexisting proteomically-distinct

164 regions and heterogeneous histological subtypes. MEpurple also positively correlated with homogeneous

165 TN regions, however, it correlated with Grade 2 tumors and high proteomic heterogeneity, but negatively

166 correlated with Grade 3, and ERPR homogeneous tumors (Fig. 4E; Extended Data Fig. 6B). Interestingly,

167 this module was enriched for leukocyte-mediated immunity and included multiple immunosuppressive

168 proteins, and checkpoint proteins, such as CD81, CEACAM1, and CD59 (Fig. 4E; Extended Data Fig.6B).

169 Thus, low grade, and specifically, Grade 2 homogeneous TN tumors may harbor immunosuppressive

170 signals, that potentially retain high proteomic heterogeneity.

In agreement with the unsupervised WGCNA, a supervised comparison between heterogeneous

172 and homogeneous tumors, while controlling for major confounding factors such as tumor grade and

173 histological subtype showed that homogeneous grade 2 ERPR IDC-NST tumors present high immune-

174 related proteins (Stat, IFIT, HLA) in the homogeneous ERPR+ tumors compared to heterogeneous ones,

175 while regions from heterogeneous tumors presented higher levels of metabolic enzymes, related to

176 glutathione metabolism, gluconeogenesis, and proline biosynthesis (Extended Data Fig.7A, C). A parallel

177 analysis of TN regions showed higher proliferation-related proteins in the homogeneous tumors, including

178 MCM complex proteins, while key TN markers (e.g., KRT5, EGFR) did not significantly differ according to 
the heterogeneity state (Extended Data Fig.7B-D). Of note, heterogeneous TN tumors also expressed markers of luminal subtypes (KRT8, KRT18, MUC1), despite being truly triple-negative according to immunohistochemistry (Extended Data Fig.7C-E). These results reinforce our findings that distinct pathways dominate homogeneous ERPR and TN tumors, while heterogeneous tumors of both ERPR+ and TN subtypes showed high proteomic similarity, with elevated expression of proteins related to glutathione metabolism and proline biosynthesis (Extended Data Fig.7B). Altogether, our results present the complex, multilayered heterogeneity and its impact on tumor progression and highlight the importance of broadening the current clinical classification in order to improve therapeutic potential.

\section{Discussion}

Recent studies highlighted the necessity and urgency of a global tumor characterization approach in

clinical practice by integrating different levels of information, especially for highly heterogeneous

tumors $^{19-21}$. Our proteomic analysis of histopathologically-defined regions created a multidimensional resource, which enables association between clinical features, spatial localization, and the proteomic landscapes in a systematic way. Using the supervised multi-region approach we demonstrated the advantage of capturing subtype-specific information and by adding spatial information of these regions, we found novel intratumor changes, among them, immune infiltrated tumor margins in TN regions, and elevated cell matrix adhesion in peripheral HR+ regions. These results might point at subtype-specific interactions within the tumor microenvironment ${ }^{22-24}$.

197 Our analyses identified high proteomic heterogeneity even in tumors that are otherwise defined as 198 homogeneous based on histopathological parameters. Furthermore, the proteomic signals associated with tumor progression were found to markedly vary within single tumors, despite being defined as the

200 same tumor grade. Interestingly, we found low correlation between the proteomic heterogeneity and the 201 molecular subtype heterogeneity, suggesting that current clinical diagnostics may mask critical 
subtype-dependent, dominated by cell proliferation and antigen processing and presentation, in triple-

206 highlight potential critical regulations of tumor diversification and selection. In addition, our results

207 revealed that TN regions from heterogeneous tumors have shared features with HR+ ones, suggesting

208 that the microenvironment of these regions plays a more dominant role in shaping the proteome than

209 the receptor expression level; these results testify to the risk of misdiagnosis when based on single needle

210 biopsies ${ }^{25,26}$. In agreement, HR-negative cells, which express basal markers, within HR+ tumors were

211 shown to confer resistance to standard endocrine and chemotherapies ${ }^{27}$.

212 Altogether, this study is the first to associate between the regional proteomic data and regulation of

213 tumor heterogeneity, the involvement of the immune system, and intrinsic proliferative signals, in the

214 context of the well-established breast cancer subtypes. In addition, our study can serve as a starting point

215 for future basic and translational research. We envision that similar to targeting clonal mutations,

216 targeting homogeneously-expressed proteins can be critical in reaching complete therapeutic responses.

217 Furthermore, clinical assessment of tumor heterogeneity will be able to drive treatment decision-making, 218 and will ultimately improve patient survival. 


\section{Refernces}

1 Ahn, S. et al. Genomic profiling of multiple breast cancer reveals inter-lesional heterogeneity. $\mathrm{Br}$ J Cancer 122, 697-704, doi:10.1038/s41416-019-0713-1 (2020).

2 Yates, L. R. et al. Subclonal diversification of primary breast cancer revealed by multiregion sequencing. Nat Med 21, 751-759, doi:10.1038/nm.3886 (2015).

3 Caravagna, G. et al. Detecting repeated cancer evolution from multi-region tumor sequencing data. Nat Methods 15, 707-714, doi:10.1038/s41592-018-0108-x (2018).

4 Buczak, K. et al. Spatial Tissue Proteomics Quantifies Inter- and Intratumor Heterogeneity in Hepatocellular Carcinoma (HCC). Mol Cell Proteomics 17, 810-825, doi:10.1074/mcp.RA117.000189 (2018).

5 Turashvili, G. \& Brogi, E. Tumor Heterogeneity in Breast Cancer. Front Med (Lausanne) 4, 227, doi:10.3389/fmed.2017.00227 (2017).

6 Baek, M., Chang, J. T. \& Echeverria, G. V. Methodological Advancements for Investigating Intratumoral Heterogeneity in Breast Cancer at the Bench and Bedside. J Mammary Gland Biol Neoplasia 25, 289-304, doi:10.1007/s10911-020-09470-3 (2020).

$7 \quad$ Krug, K. et al. Proteogenomic Landscape of Breast Cancer Tumorigenesis and Targeted Therapy. Cell 183, 1436-1456 e1431, doi:10.1016/j.cell.2020.10.036 (2020).

8 Tyanova, S. et al. Proteomic maps of breast cancer subtypes. Nat Commun 7, 10259, doi:10.1038/ncomms10259 (2016).

9 Yanovich, G. et al. Clinical Proteomics of Breast Cancer Reveals a Novel Layer of Breast Cancer Classification. Cancer research 78, 6001-6010, doi:10.1158/0008-5472.CAN-18-1079 (2018).

10 Shenoy, A. et al. Proteomic patterns associated with response to breast cancer neoadjuvant treatment. Mol Syst Biol 16, e9443, doi:10.15252/msb.20209443 (2020).

11 Johansson, H. J. et al. Breast cancer quantitative proteome and proteogenomic landscape. Nat Commun 10, 1600, doi:10.1038/s41467-019-09018-y (2019).

12 Kovac, B., Makela, T. P. \& Vallenius, T. Increased alpha-actinin-1 destabilizes E-cadherin-based adhesions and associates with poor prognosis in basal-like breast cancer. PLoS One 13, e0196986, doi:10.1371/journal.pone.0196986 (2018).

13 Liu, H. et al. Fascin actin-bundling protein 1 in human cancer: promising biomarker or therapeutic target? Mol Ther Oncolytics 20, 240-264, doi:10.1016/j.omto.2020.12.014 (2021).

14 Mayer, E. L. \& Krop, I. E. Advances in targeting SRC in the treatment of breast cancer and other solid malignancies. Clin Cancer Res 16, 3526-3532, doi:10.1158/1078-0432.CCR-09-1834 (2010).

15 Thu, K. L., Soria-Bretones, I., Mak, T. W. \& Cescon, D. W. Targeting the cell cycle in breast cancer: towards the next phase. Cell Cycle 17, 1871-1885, doi:10.1080/15384101.2018.1502567 (2018).

16 Roeten, M. S. F., Cloos, J. \& Jansen, G. Positioning of proteasome inhibitors in therapy of solid malignancies. Cancer Chemother Pharmaco/ 81, 227-243, doi:10.1007/s00280-017-3489-0 (2018).

17 Nicolau, M., Levine, A. J. \& Carlsson, G. Topology based data analysis identifies a subgroup of breast cancers with a unique mutational profile and excellent survival. Proc Natl Acad Sci U S A 108, 7265-7270, doi:10.1073/pnas.1102826108 (2011).

18 Morris, E. K. et al. Choosing and using diversity indices: insights for ecological applications from the German Biodiversity Exploratories. Ecol Evol 4, 3514-3524, doi:10.1002/ece3.1155 (2014).

19 Coenegrachts, L. et al. Mutation profile and clinical outcome of mixed endometrioid-serous endometrial carcinomas are different from that of pure endometrioid or serous carcinomas. Virchows Archiv 466, 415-422, doi:10.1007/s00428-015-1728-5 (2015).

20 Ramon, Y. C. S. et al. Integrating clinical, molecular, proteomic and histopathological data within the tissue context: tissunomics. Histopathology 75, 4-19, doi:10.1111/his.13828 (2019). 
Tang, W. et al. Integrated proteotranscriptomics of breast cancer reveals globally increased protein-mRNA concordance associated with subtypes and survival. Genome Medicine 10, 94, doi:10.1186/s13073-018-0602-x (2018).

26822 Tanaka, N. et al. Histological growth pattern of and alpha-actinin-4 expression in thyroid cancer. Anticancer Res 34, 3157-3163 (2014). Kondo, H. et al. Single-cell resolved imaging reveals intra-tumor heterogeneity in glycolysis, transitions between metabolic states, and their regulatory mechanisms. Cell Rep 34, 108750, doi:10.1016/j.celrep.2021.108750 (2021).

24 Jorgovanovic, D., Song, M., Wang, L. \& Zhang, Y. Roles of IFN-gamma in tumor progression and regression: a review. Biomark Res 8, 49, doi:10.1186/s40364-020-00228-x (2020). Heterogeneity and Ongoing Evolutionary Dynamics in Lung Tumors. Cell Rep 29, 2164-2174 e2165, doi:10.1016/j.celrep.2019.10.045 (2019). sequencing. N Engl J Med 366, 883-892, doi:10.1056/NEJMoa1113205 (2012). Kabos, P. et al. Cytokeratin 5 positive cells represent a steroid receptor negative and therapy resistant subpopulation in luminal breast cancers. Breast Cancer Res Treat 128, 45-55, doi:10.1007/s10549-010-1078-6 (2011). 


\section{Methods}

\section{Clinical sample collection and tumor mapping}

286 Formalin-fixed paraffin-embedded (FFPE) tissue samples were retrospectively collected from 37 breast cancer patients with primary tumors, with no pretreatment and various pathological characteristics. All samples were collected from the Institute of Pathology at the Sheba Medical Center and from the Israel National Biobank for Research (MIDGAM). The use of these samples for research was approved by the Institutional Review Board of Sheba Medical Center (approval SMC-7509-09) and the Tel Aviv University ethics committee, in accordance with the Declaration of Helsinki ethical guidelines. Tumor sections from five patients with hormone receptor-positive and triple negative breast cancer diagnosis were obtained from the MIDGAM according to protocol no. 130-2013, following approval by Ministry of Health. Clinical samples and clinical data were collected upon patient informed consent for collection, storage and distribution of samples and data for use in future research studies.

To map the histopathological characteristics of each tumor region, stained slides were assessed by trained breast pathologists from the Sheba Medical Center and receptor staining status was determined based on the Allred scoring system. To assess histological grade, H\&E-stained slides were analyzed based on Elston and Ellis semi-quantitative method. Combining these scores, we created histopathological maps and defined distinct tumor regions according to the following criteria: receptor expression (per region),

301 histological subtype (per region), and tumor grade (per region). These provide the visual representation 302 of the intratumoral heterogeneity for downstream processing by laser capture microdissection (LCM). The tumor region cohort included invasive ductal carcinoma of no special type (IDC NST) of grade 1 ( $n=10)$, grade 2 ( $n=101)$ and grade3 ( $n=81)$, invasive lobular carcinoma (ILC) of grade 2 ( $n=29)$, pleomorphic 305 carcinoma of grade $2(n=27)$, micropapillary carcinoma of grade $2(n=37)$, micropapillary carcinoma of grade $3(n=22)$, tubular carcinoma of grade $2(n=10)$, tubular carcinoma of grade $3(n=4)$, papillary 
"in situ" ( $n=14)$ and normal glands (normal breast tissue) derived from tumor-free incision margin or contralateral mammary gland $(n=19)$. Tumor regions defined based on receptor expression included: triple negative $(T N)(n=98)$, hormone receptor-positive $(H R+)(n=195)$, and HER2+ regions $(n=18)$. All

311 tumor-containing paraffin blocks were collected from every patient. The criteria for selection of regions

312 for further processing was defined as $70 \%$ enrichment of cancer cells (within a region) with a requirement 313 of minimal size of the target regions to provide sufficient material for proteomics (minimum $8 \mathrm{~mm} 2$ ).

314 Detailed clinical information of each patient is included in Supplementary Table 1A-B. Disease-free survival 315 was calculated from the date of primary treatment to the date of relapse or till the patient's death.

\section{Immunohistochemistry (IHC) staining}

317 Five consecutive $3.5 \mu \mathrm{m}$ tissue sections were sliced from all cancer-containing paraffin blocks from each patient (average 4-5 blocks per patient), mounted on positively charged glass slides, and dried overnight at $37^{\circ} \mathrm{C}$. Slides were mapped by staining with $\mathrm{H} \& \mathrm{E}$, anti-ER (DAKO), anti-PR (DAKO), anti-HER2 (DAKO), and anti-cytokeratin (Cell Marque). Staining was performed on the BOND-RX automated staining platform manually using a standard protocol.

\section{Image analysis}

324 Stained slides were scanned using the Leica Aperio VERSA Digital Pathology Scanner (Aperio Technologies

325 Inc.). Staining quantification of all selected regions was performed using the eSlide Manager software via 326 the Aperio image analysis algorithms: Nuclear (ER, PR), Membrane (HER2), and Cytoplasmic (CK). Regions 327 were defined by a pathologist and manually annotated in the software. Regions were considered to be 328 positive for the antibody staining if they reached a cutoff threshold of $10 \%$ and defined as high-intensity 329 staining (+3). Immune cell infiltration per region was quantified by modified nuclear algorithm via the eSlide Manager software. 


\section{Laser capture microdissection (LCM)}

332 PEN-membrane glass slides were used to allow combined UV and IR laser microdissection. Eight $\mu \mathrm{m}$ 333 sections were mounted on the slides, dried and deparaffinized with xylene, followed by a series of graded 334 ethanol. Staining was performed with the Paradise Plus system (Thermo Fisher Scientific). To obtain 335 sufficient protein amounts for downstream analyses, we selected a minimum tissue area of $8 \mathrm{~mm} 2$ for 336 each region of interest (corresponding to approximately 13,000 cells and $2 \mu \mathrm{g}$ protein). Stained tumor 337 samples were microdissected using the ArcturusXT laser capture microdissection system (Thermo Fisher 338 Scientific). LCM system allowed us to harvest relatively pure target populations of cancer cells using precise ultraviolet (UV) and infrared (IR) lasers under direct microscopic visualization.

\section{Proteomics sample preparation}

Microdissected tumor sections were collected in Eppendorf LoBind microcentrifuge tubes, lysed, and digested using two different protocols, using trifluoroethanol (TFE) or SDS in two sample batches. Protocol I included lysis with 50\% 2,2,2- TFE in $50 \mathrm{mM}$ ammonium bicarbonate buffer, the samples were boiled at

$34495^{\circ} \mathrm{C}$ for $1 \mathrm{~h}$, sonicated for 10 cycles in a Bioruptor sonicator (Diagenode), and then centrifuged at 17000 $345 \mathrm{xG}$ for $20 \mathrm{~min}$ to pellet tissue debris. Lysates were transferred to fresh LoBind tubes, reduced with $5 \mathrm{mM}$ dithiothreitol (DTT), and alkylated with $15 \mathrm{mM}$ iodoacetamide (IAA) for 30 minutes, followed by overnight

347 "in-solution" digestion using LysC-trypsin mix as described ${ }^{1}$. Protocol II included lysis in $4 \%$ Sodium 348 dodecyl sulfate (SDS) in $25 \mathrm{mM}$ HEPES ( $\mathrm{pH}=8$ ) lysis buffer, incubation for $1.5 \mathrm{~h}$ at $95^{\circ} \mathrm{C}$, reduction, 349 alkylation, and digestion following the Single Pot Solid Phase Sample Preparation (SP3) protocol ${ }^{2}$. SeraMag 350 Hydrophilic and Hydrophobic beads (GE Healthcare) mix was used at a concentration of $100 \mu \mathrm{g} / \mu \mathrm{l}$ for overnight "on-bead" digestion using LysC-trypsin mix (Promega) ${ }^{2,3}$. Tryptic peptides from 363 regions

352 were labeled using 10plex TMT reagent and assembled into 41 TMT-labeled sample sets. Each set was composed of nine channels for the different regions and one channel for an internal standard. The internal 
fractionated offline into eight fractions using high $\mathrm{pH}$ reverse-phase chromatography (PierceTM High pH followed by LC-MS analysis.

\section{LC-MS-based proteomics}

LC-MS/MS analysis was performed using high-performance liquid chromatography (Easy nLC 1000 HPLC system) coupled on-line to a Q-Exactive HF mass spectrometer (Thermo Fisher Scientific) through the columns (Thermo Fisher Scientific) and loaded with Buffer A ( $0.1 \%$ formic acid). Peptides were eluted with a 140-minute gradient of water-acetonitrile, and each batch of samples, composed of eight factions, was analyzed in a total of 20 hours. We slightly modified the gradients for each fraction as follows: for fractions 1 and 2, 5-30\% buffer B (80\% acetonitrile/0.1\% formic acid); for fraction 3, 5-31\% buffer B; for fraction 4,

B. All fractions were eluted at a flow rate of $200 \mathrm{nl} / \mathrm{min}$ at $40^{\circ} \mathrm{C}$. MS data were acquired in a datadependent mode and the acquisition method included a full scan event at a resolution of 120,000 , followed by top $10 \mathrm{MS} / \mathrm{MS}$ scans at a resolution of 60,000 .

\section{Data Processing: proteomics raw MS data processing}

371 Raw MS files were analyzed with the MaxQuant software (version 1.6.2.6) and the Andromeda search

372 engine ${ }^{4}$. The Uniprot database (human, version from 2018 with 95057 entries) was used for searching 373 MS/MS spectra. Modifications for 10plex TMT labels were defined in MaxQuant and included the label 374 impurities as indicated for each label batch. A decoy database was used to determine a $1 \%$ false discovery 375 rate for both protein identification and peptide spectrum matches. We included carbamidomethyl 376 cysteine as a fixed modification and $\mathrm{N}$-terminal acetylation and methionine oxidation as variable 377 modifications with a maximum of five modifications per peptide. Maximum two missed cleavages were 378 allowed. 


\section{Proteomic statistical analysis}

380 All statistical tests were performed using Perseus, Python, and R. We used the proteinGroups output table

381 for the analysis (Supplementary Table 2). Initial data processing included filtration of proteins originating

382 from the decoy database (reverse proteins), proteins only identified by modification sites and common

383 contaminants. In addition, we filtered out extracellular matrix proteins to avoid distortion of the overall

384 ratio distribution by high and variable expression of extracellular proteins. The 33 regions that had less

385 than 3000 protein IDs were excluded from the analysis. Protein expression values (reporter intensities

386 corrected) were log2-transformed and normalized to the internal control in each TMT set. Then the entire

387 dataset was filtered to retain proteins with a minimum of $70 \%$ of valid values, reaching 4046 protein

388 groups, which were used for most downstream analyses. The data were further zero-centered by

389 subtracting the median of each sample. We imputed missing values by creating an artificial normal

390 distribution from the missing values, with a downshift of 1.9 standard deviations and a width of 0.5 of the

391 original ratio distributions, and after that, applied quantile normalization algorithm using $\mathrm{R}$ code from the

392 "Biobase" package. The entire cohort was analyzed in four batches, including two sample preparation

393 procedures, as described above. To correct the batch effect of the two sample preparation procedures,

394 we subtracted the first component in a Principal Component Analysis. For supervised analyses, all

395 normalizations were performed on the selected group of samples included in each specific analysis. This

396 allowed maximizing the number of proteins included in each analysis. Normalizations included Log2-

397 transformation, normalization to the internal control, filtration to retain proteins with at least $70 \%$ valid

398 values, and zero-centering.

399 Specific statistical analyses were performed as follows:

400 - Analysis of pseudo-bulk samples was performed by calculating the median expression of each

401 protein from regions of the same tumor. Subtype annotations for the bulk tumors were based on the

402 clinical reports of the whole tumor. For comparison of keratin expression levels between pseudo-bulk and 
region data, we performed Kruskal-Wallis test and created boxplots using "ggpubr" package in R.

404 Significantly differentially expressed proteins between pseudo-bulk subtypes were extracted by performing Welch's test with Benjamini-Hochberg (BH) FDR of 0.1. In order to compare the results with multi-sampled regions and correct for the significant differences in sample size, Welch's test (BH FDR 0.1) was performed by drawing random samples (the same number of samples as in the pseudo-bulk analysis) from the dataset. The random sampling was repeated 100 times and the number of significantly different proteins was annotated.

- Unsupervised clustering was performed using modified minimum variance method ('ward.D2').

411 For the analysis, we used non-imputed matrix with $100 \%$ valid values and calculated Euclidean distances

412 between regions. Then applied the modified Ward's method to create groups with minimized variance

413 within clusters. To represent our cohort of 330 regions, we used Tree-and-Leaf dendrogram where each

414 node represents a tumor region, branches of the tree constructed based on the Euclidean distances and 415 cluster separation presented by spatial localization of the branches. For visualization we used R code from 416 the "TreeAndLeaf" package.

417 - Supervised analysis based on subtype and grade was performed using one-way ANOVA followed 418 by post Hoc tests with permutation-based FDR cutoff of 0.05 using the Perseus platform. Results were 419 visualized in R using the 'ComplexHeatmap' package. Enrichment analyses were performed using Fisher 420 exact test with an FDR cutoff of 0.05 , and the entire dataset of 8150 proteins as background. To 421 demonstrate variation of malignancy scores we used the "ggplot2" package in R for visualization.

422 Canonical correlation analysis (CCA) was performed to reduce dimensionality of the proteomics 423 data. First, we used scikit-learn PCA in Python to reduce the data dimension to 240 components, which 424 explained $>99 \%$ of the variance. Second, we performed canonical correlation analysis of the proteomics 425 and clinical data, further reducing the data to its 7 canonical correlation components. For the analysis, we 
427 visualization of the CCA results, we plotted 3D scatterplots using "scatterplot3d" package in R. To show

428 the data hierarchy, we used absolute Spearman rank correlation analysis between canonical components

429 and pathological features, and visualized it using R code from the "pheatmap" package.

- $\quad$ Spatial distance calculation was performed by measuring the physical distance from the tumor

431 margin to each region of the same tumor. To obtain measurements, we used the eSlide Manager software

432 via the Aperio image analysis tools (ruler). First, we calculated the absolute spatial distance (absSpaD), 433 and then normalized each distance to the tumor size to construct normalized spatial distance (nSpaD) to 434 compare across tumors.

435 absSpaD $=1 / 2 \varnothing-$ avD

436 where $1 / 2 \varnothing$ corresponds to the half of the measured tumor diameter and avD is the average distance from

437 the tumor margin

438 Normalized spatial distance (nSpaD) is the distance from the tumor center, normalized to the tumor size:

$\mathrm{nSpaD}=1-(\mathrm{absSpaD} / \mathrm{tS})$

440 where tS corresponds to the tumor size.

$441 \quad$ Stable and variable proteins calculation was calculated as the median absolute deviation (MAD)

442 scores per protein. Based on the MAD score we ranked every protein per tumor. Using sum of ranked

443 proteins, we selected top 5\% for stable and variable group and performed functional enrichment. We

444 visualized the output in Cytoscape 3.8.2 via the "EnrichmentMap" plugin. For the EnrichmentMap, we 445 used node FDR cutoff of 0.05 and edge cutoff (Jaccard similarity index) of 0.25 . Redundant processes were 446 removed from resulted networks. 
448 heterogeneity (ITH) scores and a malignancy score. For molecular and histological subtypes, we calculated

449 the number of coexisting subtypes per tumor, and then obtained the area measure of each subtype via

450 the eSlide Manager software. The score was calculated as

$451 \quad \mathrm{M}-\mathrm{ITH}=1-(\mathrm{F} / \mathrm{n}) \quad$ and $\quad \mathrm{H}-\mathrm{ITH}=1-(\mathrm{F} / \mathrm{n})$

452 where $F$ corresponds to the largest fraction of subtype from the total area of tumor and $n$ is number of

453 subtypes coexisting in the same tumor. Obtained scores were calculated per tumor.

454 For the proteomic ITH score, we calculated the correlations between all pairs of tumor regions using log2

455 transformed non-normalized dataset of 8150 protein groups, and then averaged the data from two non-

456 overlapping pairs with the lowest correlation per tumor to avoid any subjective effect of multisampling.

457 The score was calculated per tumor which allowed us to avoid batch effect and opt for using a whole non-

458 normalized dataset of 8150 proteins. The score was calculated as

$459 \quad \mathrm{P}-\mathrm{IHT}=1-\mathrm{avC}$ calculated per tumor.

For malignancy score calculation we applied analysis of disease progression by averaging the proteomic

463 data of normal regions of 13 patients from our cohort, and then calculated the Pearson correlation of 464 each tumor region to the normal tissue. Log2 transformed normalized data of 4046 protein groups was 465 used as input. The scores were calculated as

Malignancy (M) score $=1-\mathrm{C}(\mathrm{avN})$. calculated per region. 
471 (molecularly or histologically), in the context of the entire tumor. Each region was assessed based on its

472 subtype definition and whether its own subtype definition was different/same as the majority of the

473 tumor area ( $>70 \%$ of the area).

$474 \quad-\quad$ To create protein networks correlating to the scores, first, we filtered proteins based on their

475 Pearson correlation to each score with established cutoff of 0.2. After merging the lists, we built a protein 476 network using STRING output via Cytoscape. 1D annotation enrichment analysis was performed on the 477 score values with a Benjamini-Hochberg FDR threshold of 0.02 using Perseus software. A Chi-square test 478 was performed for comparative correlation between tumor grade and heterogeneity of the tumors using 479 R code from the "stats" package.

- Weighted gene co-expression network analysis (WGCNA) was performed using R code from the

481 "WGCNA" package using signed network, bicor correlation function, power $=10$ and a reassign threshold 482 of 0.1 . Out of 330 regions, six regions were excluded as outliers and three regions were excluded due to 483 the absence of receptor quantification data. Results of the WGCNA were visualized using "pheatmap" and

484 "WGCNA" packages in R. For enrichment analysis we performed Fisher exact test with a Benjamini485 Hochberg FDR q-value $<0.02$ using Perseus software. To validate our findings in a supervised manner, 486 while controlling for major confounding factors such as tumor grade and histological subtype, we selected 487 only Grade 2 tumors of one histological subtype (IDC-NST). A Welch's test with permutation-based FDR cutoff of 0.05 , comparing between homogeneous and heterogeneous status of each subtype was performed using Perseus software on non-imputed data. 
bioRxiv preprint doi: https://doi.org/101101/2021.08 05.455361; this version posted August 6, 2021. The copyright holder for this preprint (which was not certified by peer review) is the author/funder, who has granted bioRxiv a license to display the preprint in perpetuity. It is made available under aCC-BY-NC 4.0 International license.

490

491 using $\mathrm{R}$ code via the 'ComplexHeatmap' package. Receptor staining quantification and clinical data

492 correlations were visualized using "pheatmap", "corrgram" and "corrplot" packages in R.

\section{Data and software availability}

494 All raw data are available via ProteomeXchange with identifier PXD024190. Reviewer account 495 details: Username: reviewer_pxd024190@ebi.ac.uk; Password: xUr5MpUo 


\section{References}

4971 Shenoy, A. et al. Proteomic patterns associated with response to breast cancer neoadjuvant treatment. Mol Syst Biol 16, e9443, doi:10.15252/msb.20209443 (2020). Hughes, C. S. et al. Single-pot, solid-phase-enhanced sample preparation for proteomics experiments. Nat Protoc 14, 68-85, doi:10.1038/s41596-018-0082-x (2019).

502

503 Hughes, C. S. et al. Ultrasensitive proteome analysis using paramagnetic bead technology. Mol 3 Syst Biol 10, 757, doi:10.15252/msb.20145625 (2014).

4 Cox, J. \& Mann, M. Quantitative, high-resolution proteomics for data-driven systems biology. Annu Rev Biochem 80, 273-299, doi:10.1146/annurev-biochem-061308-093216 (2011).

Acknowledgements

507 The Geiger laboratory received Funding from the European Research Council (ERC) starting grant [639534]

508 and the Israel Science Foundation (grant 748/16). We thank Georgina Barnabas, Gali Yanovich-Arad, 509 Michal Harel, Michael Selitrennik, and the members of Geiger laboratory for the fruitful discussions and assessment of the manuscript. We thank Eilam Yeini and Professor Ronit Sachi-Fainaro for help with

511 immunohistochemistry; Dr. Adi Zundelevich for assistance with patient cohort assembly; members of the

512 Pathology Department at the Sheba Medical Center for assistance in tissue selection and processing; Israel

513 National Biobank for Research (MIDGAM) for assistance in tissue selection. We thank Constantiner

514 Institute for the partial support.

\section{Author contributions}

516 Conceptualization, T.G, and M.M; Investigation, M.M; Formal analysis, M.M, A.S, R.S, D.P, N.I and I.B; Data

517 curation, K.K, D.N, I.M and B.S; Resources, I.B and E.N.G; Writing Original Draft, M.M and T.G; Writing

518 Review \& Editing, M.M, A.S, R.S, E.N.G and T.G; Supervision, T.G; Funding acquisition, T.G.

519 Declaration of interests

520 The authors declare no conflict of interests.

\section{Materials \& Correspondence}

522 Tamar Geiger tami.geiger@weizmann.ac.il 
bioRxiv preprint doi: https://doi.org/10.1101/2021.08.05.455361; this version posted August 6, 2021. The copyright holder for this preprint (which was not certified by peer review) is the author/funder, who has granted bioRxiv a license to display the preprint in perpetuity. It is made available under aCC-BY-NC 4.0 International license.

Mardamshina et al., Figure 1
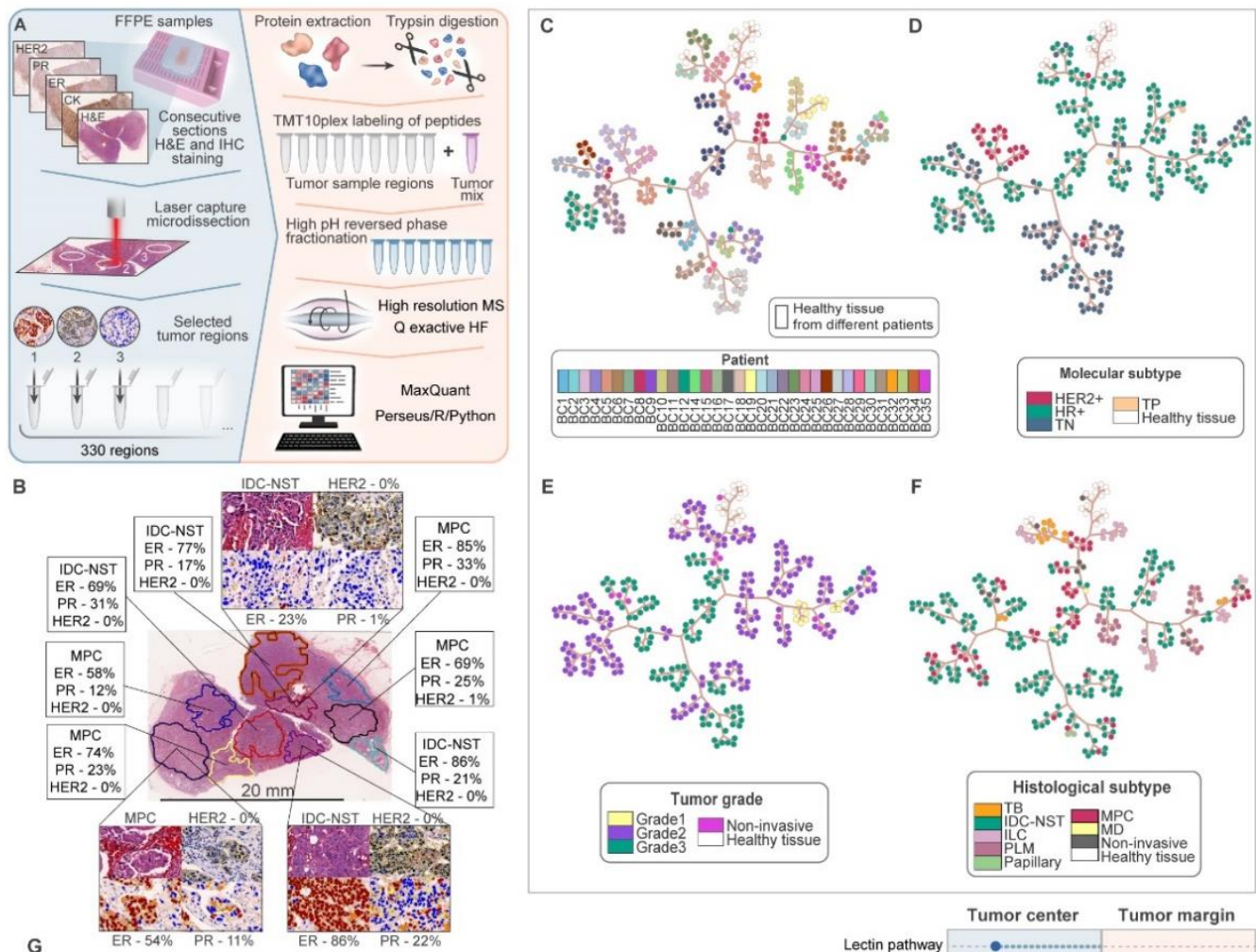

E

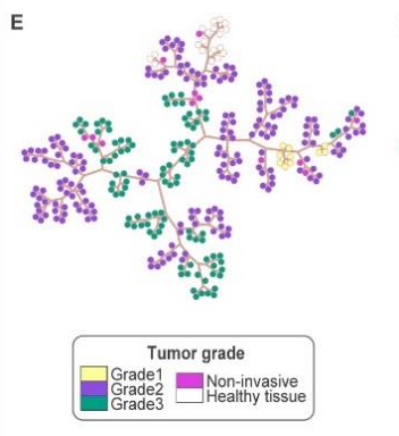

$\mathbf{F}$
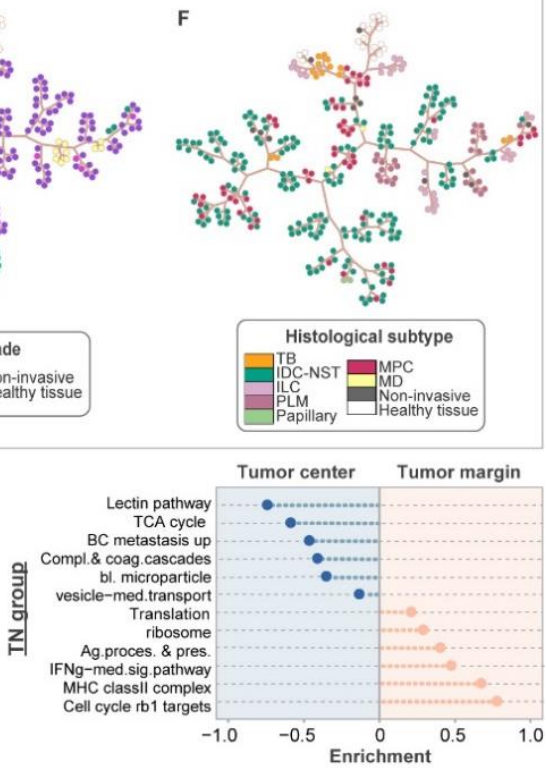

Fig. 1: Unsupervised analysis of proteomics of breast cancer

(A) Proteomic analysis was performed on FFPE tumor sections. To map the tumor histological and molecular characteristics, we performed immunohistochemistry and H\&E staining of consecutive sections and followed with laser-capture microdissection of histopathologically-defined regions from each tumor. Extracted proteins were trypsin-digested, labeled with TMT10plex, mixed with an internal standard, and separated using high pH reverse phase fractionation. Peptide samples were analyzed on the Q Exactive HF MS followed by computational analysis.

(B) Histopathological analysis of intratumor heterogeneity was based on the combination of ER, PR, HER2, and H\&E staining of consecutive sections and quantification using the eSlideManager software. BC7 is shown as an example of the divergence of ER expression and histological features among regions of the same tumor. Images of receptor staining show the overlay of quantification analysis that demonstrate different expression intensity detected by the algorithm, where brown color indicates high intensity staining, orange - moderate intensity, yellow - mild intensity, and blue - no staining.

(C-F) Tree-and-leaf representation of dendrogram based on modified minimum variance method where each node represents a tumor region, branches of the tree are constructed based on Euclidean distances and the cluster separation is presented by the spatial localization of the branches. Presented dendrograms are color-coded based on: (C) Patient identity; (D) Molecular subtype; (E) Tumor grade; and (F) Histological subtype.

(G) Schematic representation of spatial distances measurement in each tumor. Barplots show enrichment analysis of HR+ and TN tumors. 1D Enrichment analysis was performed with FDR q-value $<0.02$. 
bioRxiv preprint doi: https://doi.org/10.1101/2021.08.05.455361; this version posted August 6, 2021. The copyright holder for this preprint (which was not certified by peer review) is the author/funder, who has granted bioRxiv a license to display the preprint in perpetuity. It is made available under aCC-BY-NC 4.0 International license.

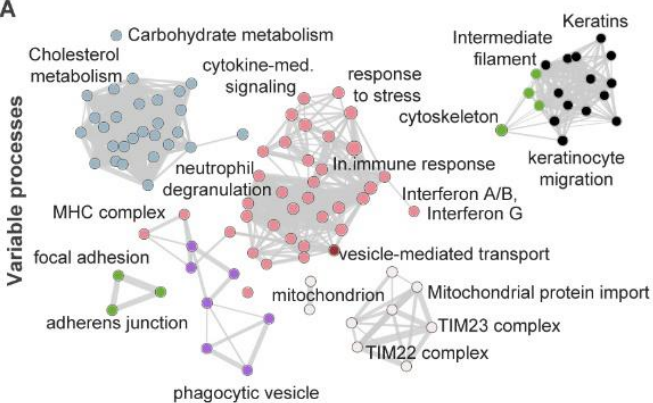

Metabolic processes Proliferation/Translation machinery Proteasome Signal transduction

Phagocytosis

Immune processes Focal adhesion/cell-cell interaction

- Keratins

B

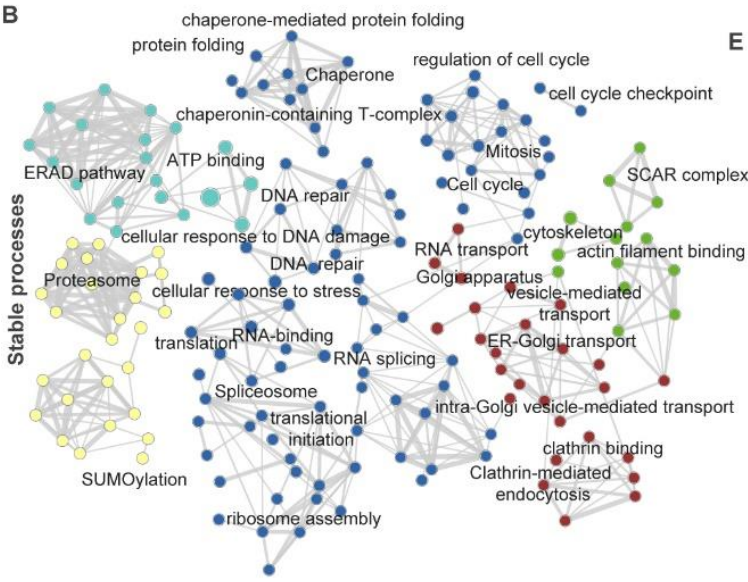

Mardamshina et al., Figure 2
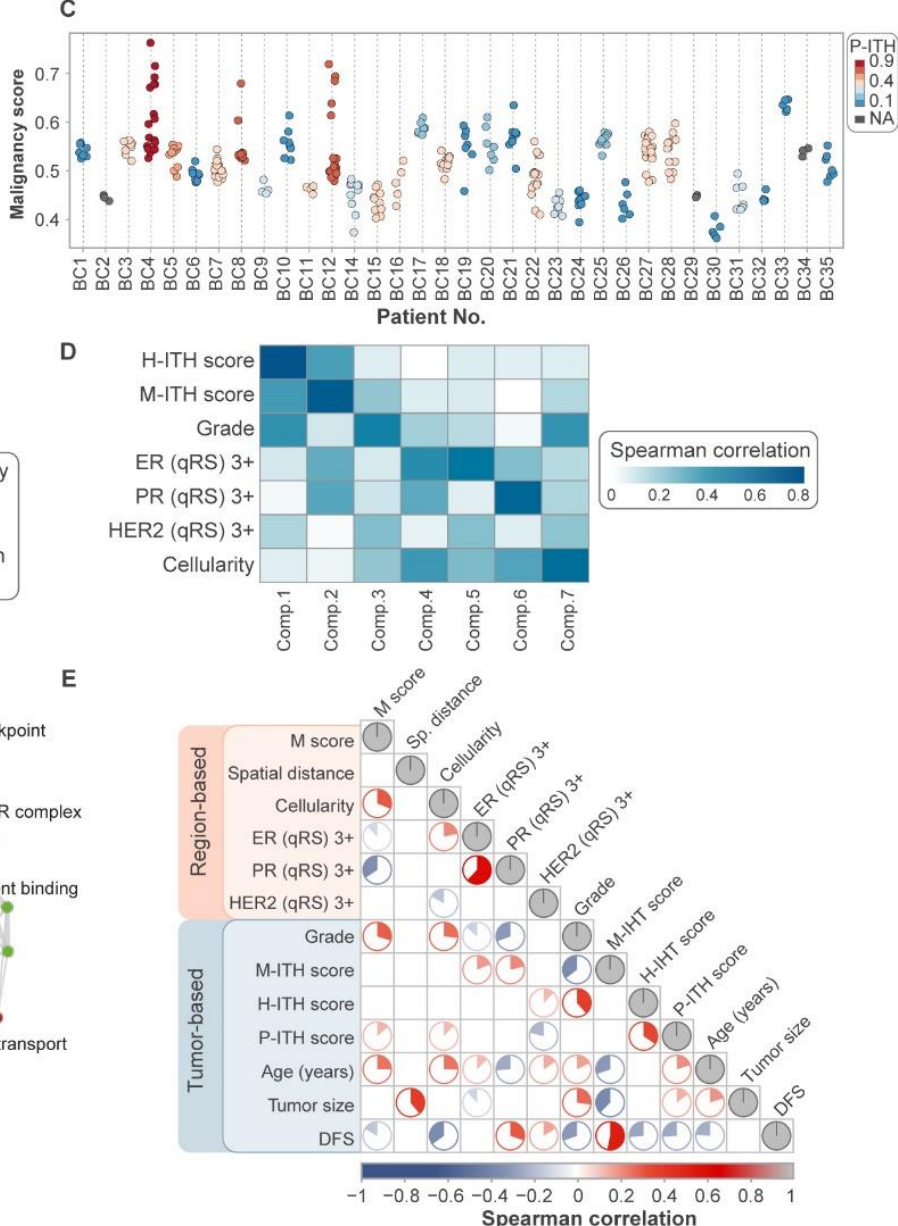

Fig. 2: Proteomic-based intratumor heterogeneity.

548 (A-B) Enrichment network analysis was performed separately for constant and variable proteins (based on the top $5 \%$ ranked proteins). It resulted in two networks with processes of variable proteins (top network) and constant proteins (bottom network) across all 35 tumors. Network nodes correspond to the enriched processes, edges represent mutual overlap.

552 (C) Dotplot shows the intratumoral diversity of regional malignancy scores and interplay between two proteomicsbased scores. Each tumor is color-coded based on its P-ITH score.

554 (D) Correlation heatmap of seven canonical components to the pathological features shows that data variance is 555 most predominantly explained by heterogeneity ( $\mathrm{H}-\mathrm{ITH}$ and $\mathrm{M}-\mathrm{ITH}$, first two components), then grade and molecular 556 subtypes. Color scheme represents the results of absolute spearman rank correlation analysis.

557

(E) Correlation heatmap of clinical (pathological) features and the calculated scores. Only the statistically significant 
bioRxiv preprint doi: https://doi.org/10.1101/2021.08.05.455361; this version posted August 6, 2021. The copyright holder for this preprint (which was not certified by peer review) is the author/funder, who has granted bioRxiv a license to display the preprint in perpetuity. It is made available under aCC-BY-NC 4.0 International license.

A

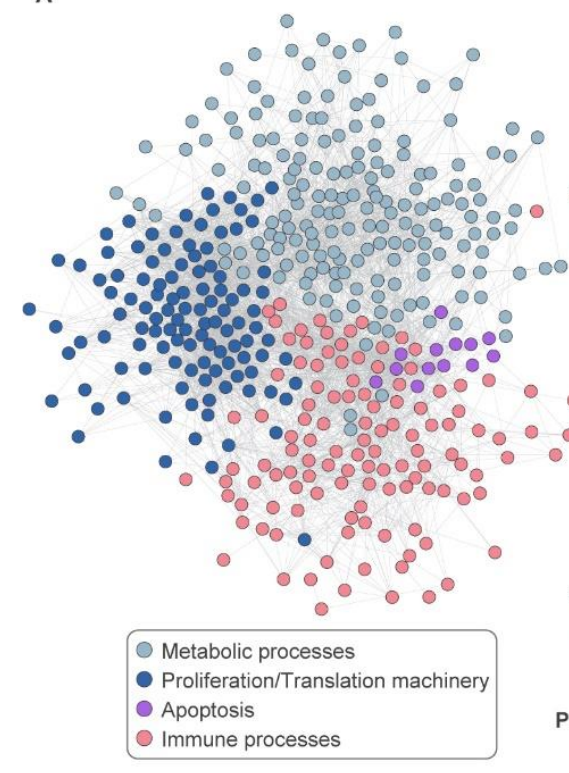

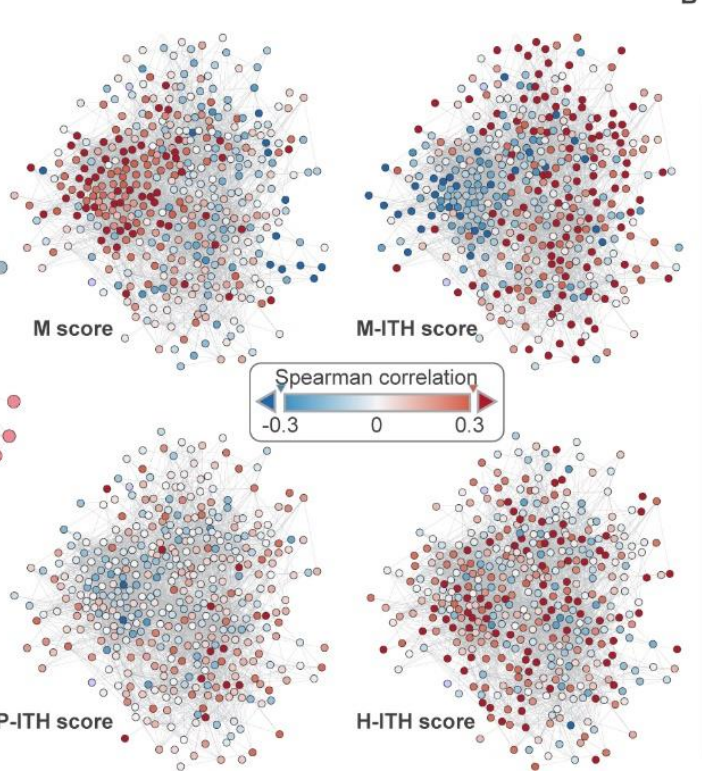

561 Fig. 3: Analysis of multi-layered intratumor heterogeneity.

562

563

564

565

566

567

568
Mardamshina et al., Figure 3

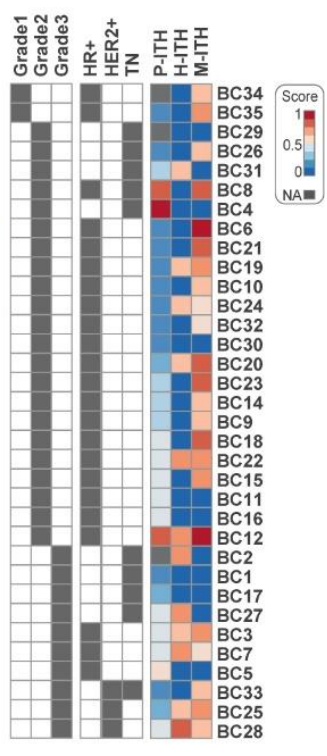

(A) Merged protein-protein interaction network of the positively correlating proteins with each of the four scores (malignancy score and the three ITH scores, Pearson correlation $>0.2$ ). On the left, the network is colored based on selected enriched annotations, and the four networks on the right are color-coded based on the correlation values with each one of the scores.

(B) Integrated heatmap shows combination of clinical parameters and internal heterogeneity scores at the tumor level shows the switch between molecular and histological ITH depending on the tumor grade. 
bioRxiv preprint doi: https://doi.org/10.1101/2021.08.05.455361; this version posted August 6. 2021. The copyright holder for this preprint (which was not certified by peer review) is the author/funder, who has granted bioRxiv a license to display the preprint in perpetuity. It is made available under aCC-BY-NC 4.0 International license.

Mardamshina et al., Figure 4

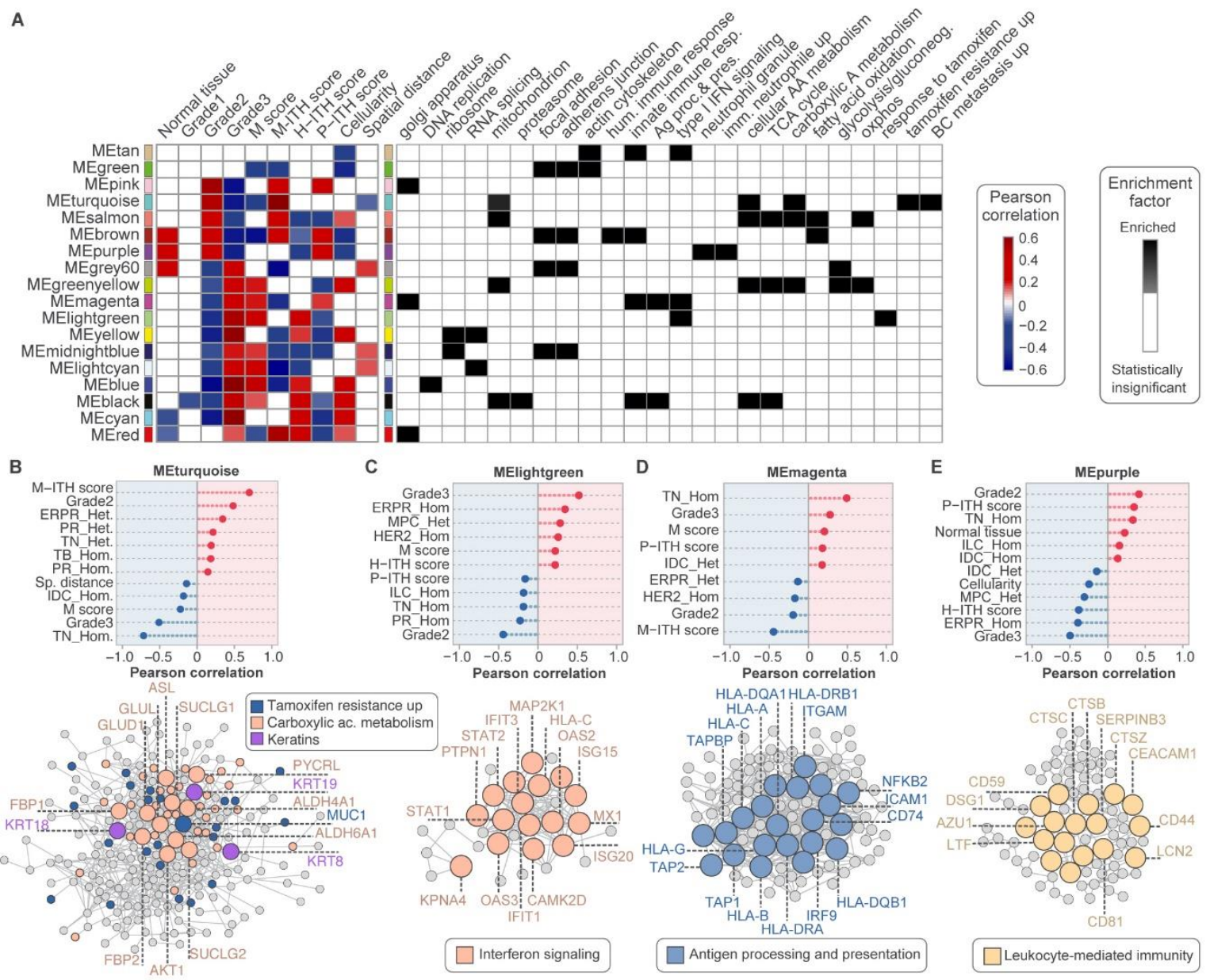

Fig. 4: Unsupervised weighted gene co-expression network analysis (WGCNA) of different levels of intratumoral heterogeneity.

(A) WGCNA of 321 regions (tumor and normal tissue) separated the proteomics data into 18 modules. The left heatmap shows the Pearson correlation of module eigengenes (MEs) with selected clinical parameters and heterogeneity scores. Only significant correlations are presented (Benjamini-Hochberg FDR q-value $<0.05$ ). The full correlation heatmap is presented in Extended Data Fig. 6. The right heatmap demonstrates functional enrichment for different MEs (Fisher exact test with Benjamini-Hochberg FDR q-value < 0.02). The statistical parameters of WGCNA are presented in Extended Data Fig. 5.

(B-E) Cleveland plots show Pearson correlations of the pathological features to selected module eigengenes: MEturquoise (B), MElightgreen (C), MEmagenta (D) and MEpurple (E). Only significant correlations are presented (Benjamini-Hochberg FDR q-value < 0.05). Protein-protein interaction networks of the modules were generated 
bioRxiv preprint doi: https://doi.org/10.1101/2021.08.05.455361; this version posted Auqust 6, 2021. The copyright holder for this preprint (which was not certified by peer review) is the author/funder, who has granted bioRxiv a license to display the preprint in perpetuity. It is made available under aCC-BY-NC 4.0 International license.

EXTENDED DATA FIGURES

Mardamshina et al., Extended Data Fig. 1

A

B

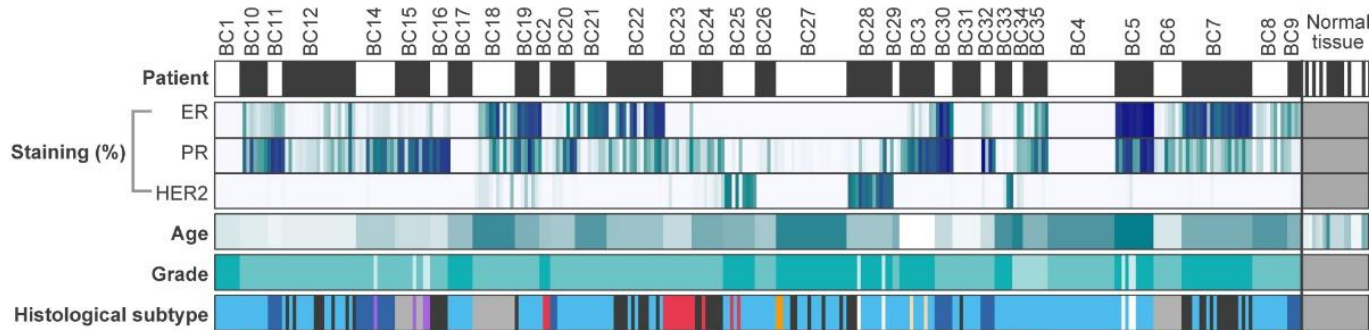

Molecular subtype

Cellularity

Tumor size

DFS

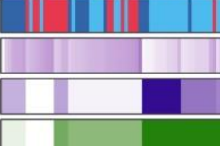

| |||||||||||||||| ||||||

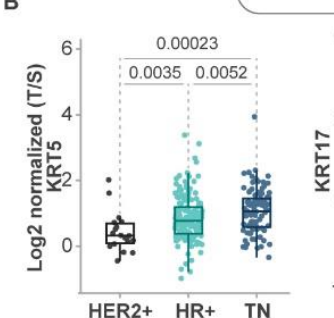

C

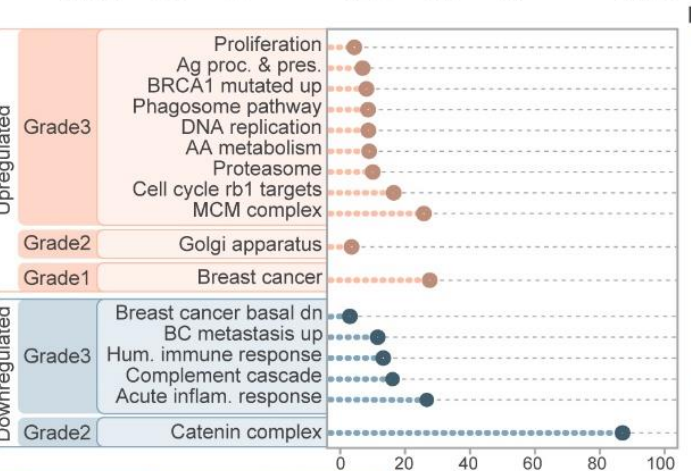

D
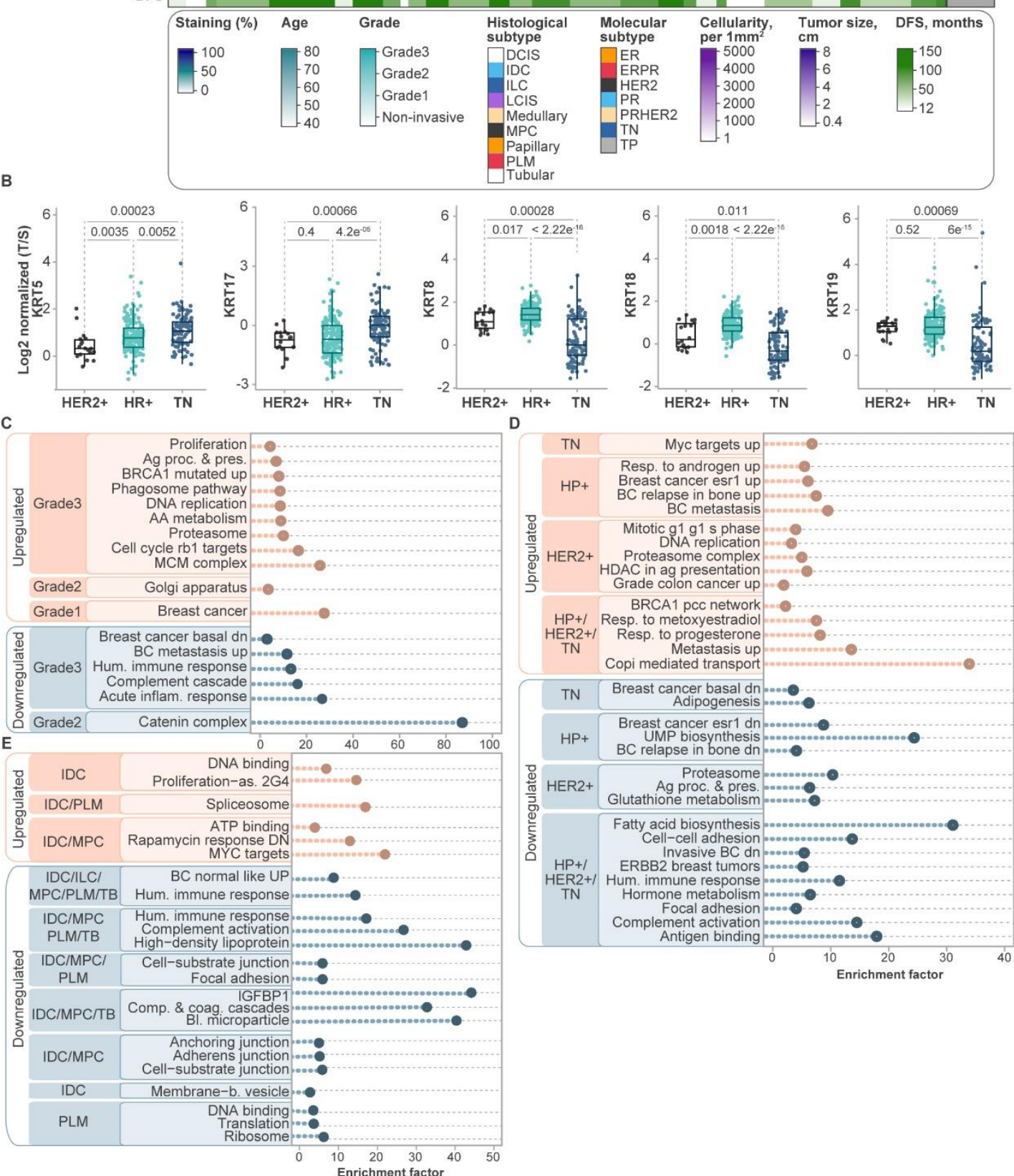
586 Extended Data Fig. 1: Clinical features of the cohort and proteomic comparison to healthy control samples.

587 (A) The study cohort includes 330 regions derived from 35 breast cancer patients. Heatmap indicates the clinical 588 parameters. Patient row separates between all patients by switching black and white bars, and all normal samples 589 in the cohort are indicated in grey.

590 (B) Boxplots demonstrate subtype specific changes of keratin expression in the different regions. Statistical 591 significance was determined with Kruskal-Wallis test, $p<0.05$. HR+ regions include ER, ERPR and PR+ regions.

592 (C) Enrichment analyses of selected processes that deviate from healthy tissue show processes associated with 593 tumor grade. The entire list of enriched processes can be found in the Supplementary Table 3A.

594 (D) Enrichment analyses of selected processes that deviate from healthy tissue show processes associated with 595 molecular subtype. The entire list of enriched processes can be found in the Supplementary Table 3A.

596 (E) Enrichment analyses of selected processes that deviate from healthy tissue show processes associated with 597 histological subtype. The entire list of enriched processes can be found in the Supplementary Table 3A. 
A
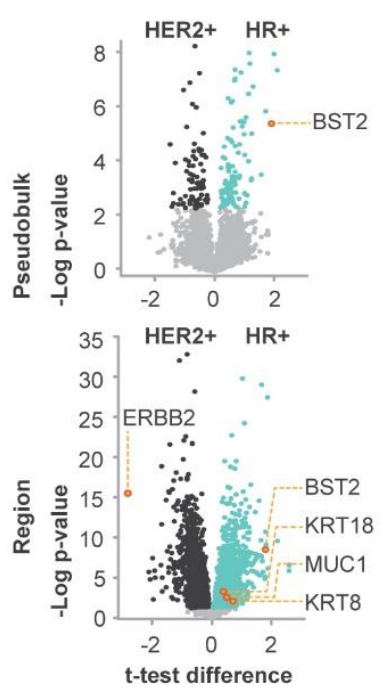
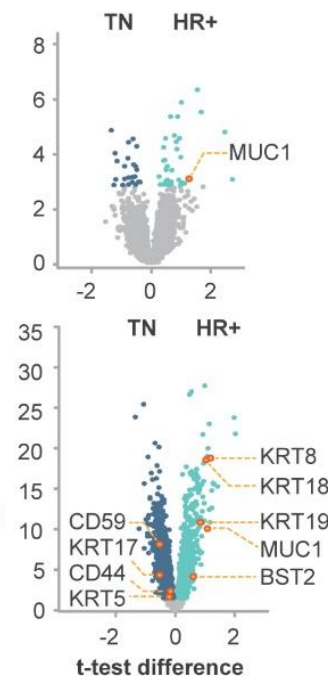

Mardamshina et al., Extended Data Fig. 2

B

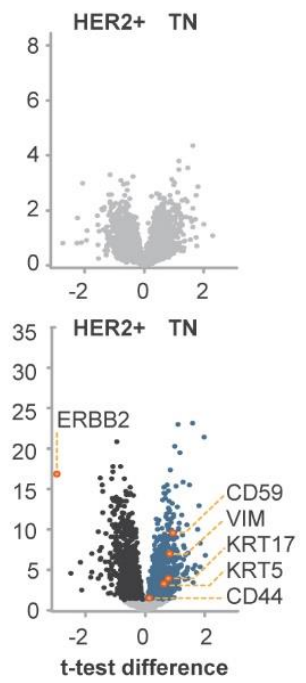

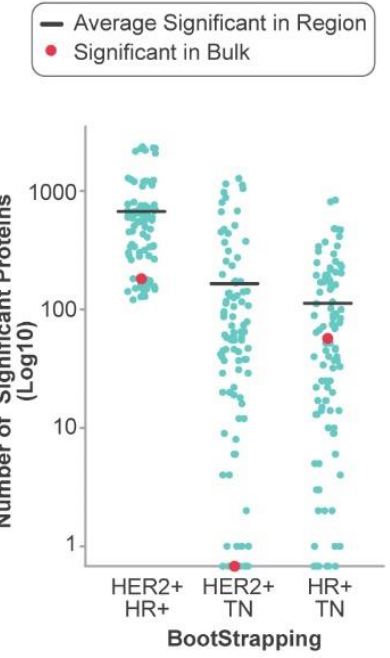

600 Extended Data Fig. 2: Advantage of multi-region approach for breast cancer analysis.

601 (A) Volcano plots show the advantage of regional data over bulk analyses in capturing subtype specific significantly 602 changing proteins. Statistical significance was determined with Welch's t-tests, with permutation-based FDR q-value $603<0.05$.

604 (B) Dotplot presents the number of significantly different proteins upon bootstrapped Welch's test (with 100 605 iterations of random sampling). The number of significantly changing proteins in the pseudo-bulk analysis is 606 indicated by a red dot. 
bioRxiv preprint doi: https://doi.org/10.1101/2021.08.05.455361; this version posted August 6, 2021. The copyright holder for this preprint (which was not certified by peer review) is the author/funder, who has granted bioRxiv a license to display the preprint in perpetuity. It is made available under aCC-BY-NC 4.0 International license.

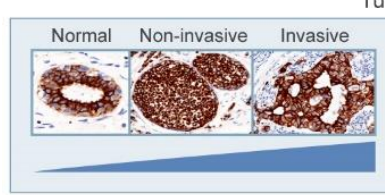

B

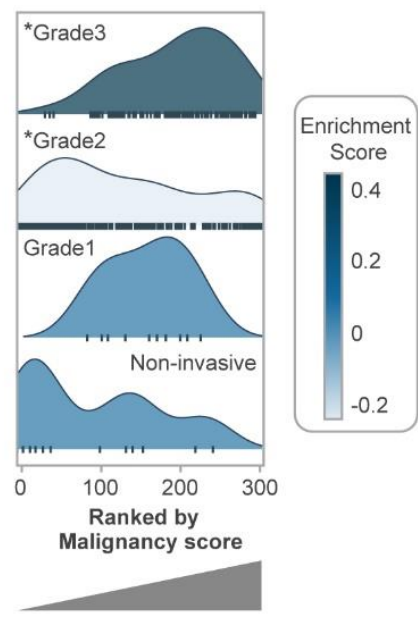

Tumor progression

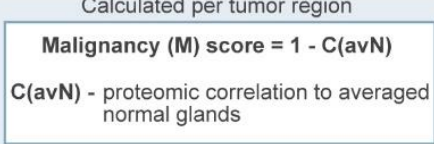

E
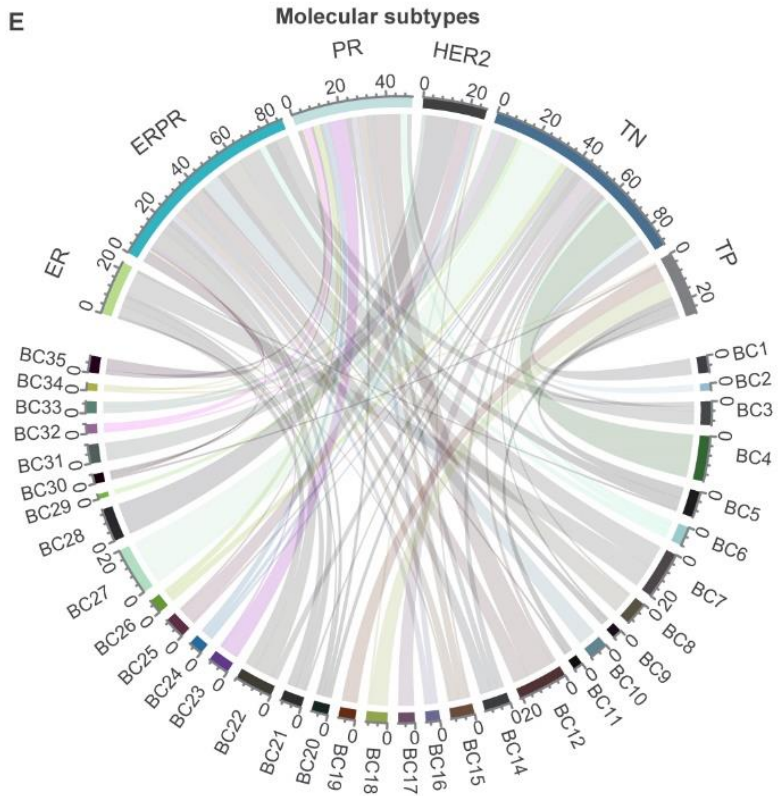

Proteomics-based scores

Mardamshina et al., Extended Data Fig. 3
C

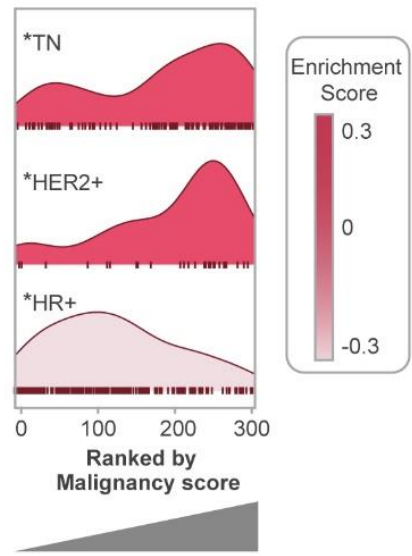

Proteomic profile

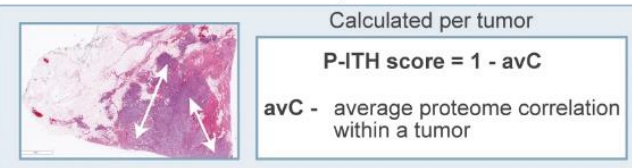

D

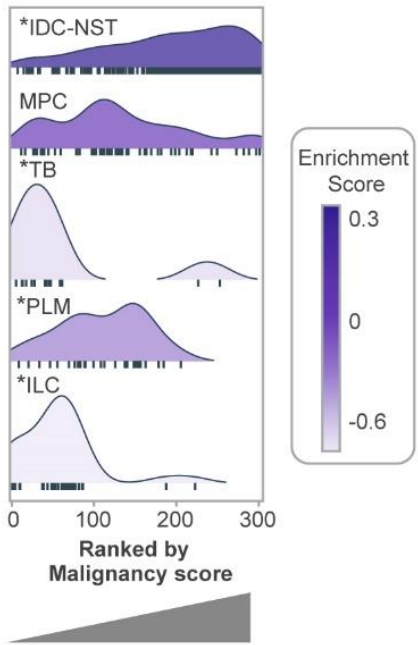

Histological subtypes

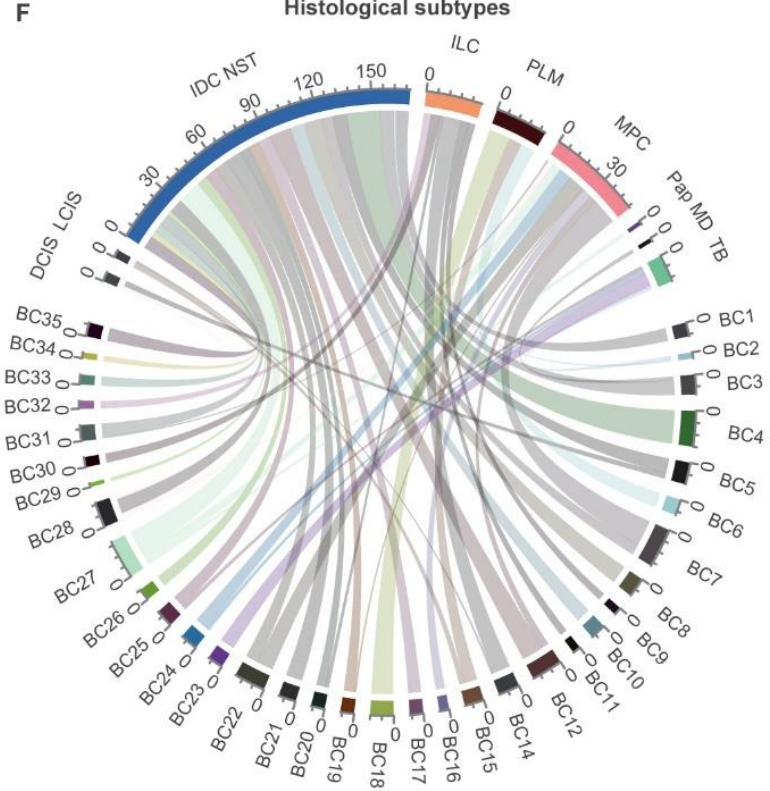

G

Pathological parameters-based scores

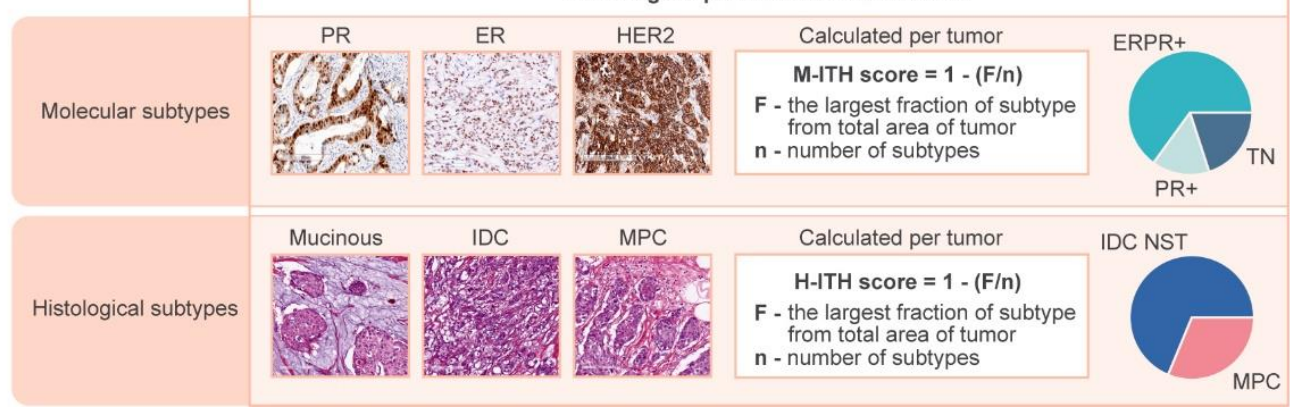


609 Extended Data Fig. 3: Analysis of intratumor heterogeneity.

610 (A) Quantitative analysis of proteomics-based intratumoral heterogeneity defined malignancy score (M) and 611 proteomic heterogeneity score $(\mathrm{P}-\mathrm{ITH})$ based on the proteomic data. $\mathrm{M}$ score is calculated per region, while $\mathrm{P}$-ITH 612 score is calculated per tumor.

613 (B-D) Ridgeline density plots demonstrate ranking of tumor regions by their malignancy scores. Higher malignancy 614 scores are presented in Grade 3 tumor regions (B), in TN and HER2 regions (C), and in IDC-NST regions (D). Grade 2 615 tumors present a wide distribution of malignancy scores. Asterisks represents FDR q-value $<0.05$.

616 (E, F) Chordplots show distribution of regions of each tumor between represented subtypes: Molecular (E) and 617 histological (F). Each tumor consists of several regions that belong to one or several subtypes. HR+ regions (ER, ERPR, 618 PR) demonstrate higher intratumoral heterogeneity than TN regions. Plots show that most tumors are composed of 619 more than one histological and more than one molecular subtype.

620 (G) Quantitative analysis of pathological intratumoral heterogeneity defined molecular subtypes heterogeneity 621 score (M-ITH) and histological subtypes heterogeneity score (H-ITH). M-ITH and H-ITH are based on clinical 622 parameters (H\&E and receptor IHC); both scores are calculated per tumor. 
bioRxiv preprint doi: https://doi.org/10.1101/2021.08.05.455361; this version posted August 6, 2021. The copyright holder for this preprint (which was not certified by peer review) is the author/funder, who has granted bioRxiv a license to display the preprint in perpetuity. It is made available under aCC-BY-NC 4.0 International license.

Mardamshina et al., Extended Data Fig. 4

A
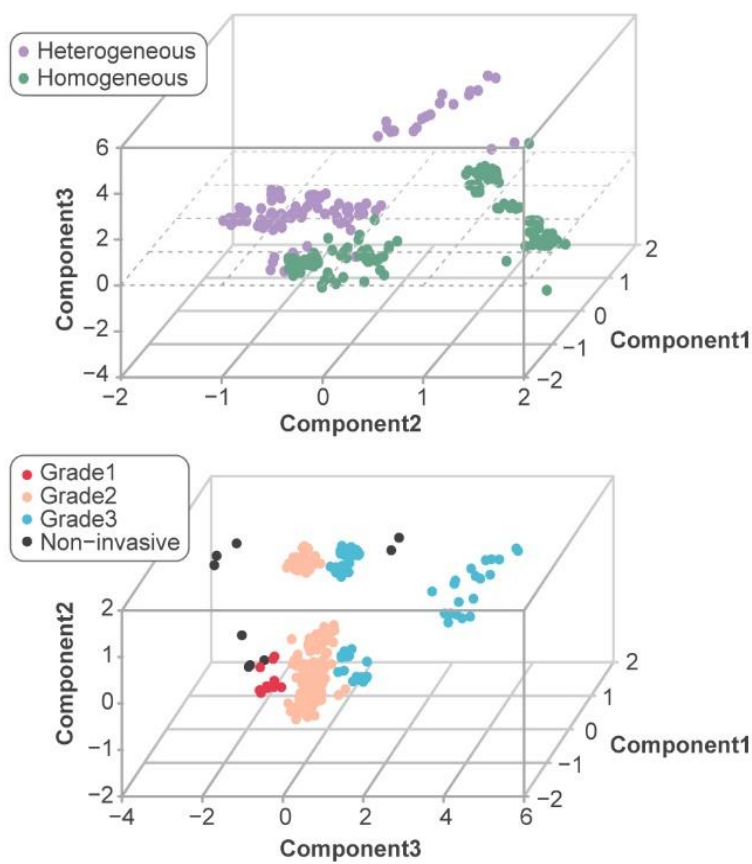

B

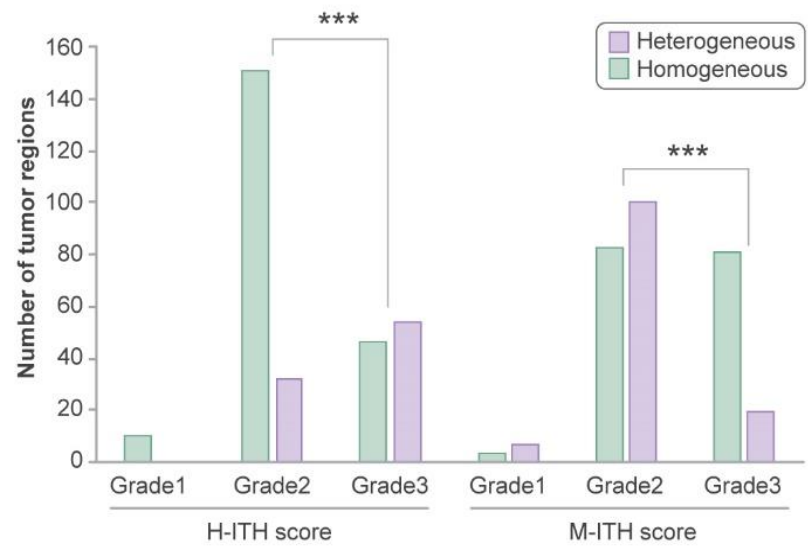

${ }^{* * *} X^{2}$ pvalue $<0.00001$
C

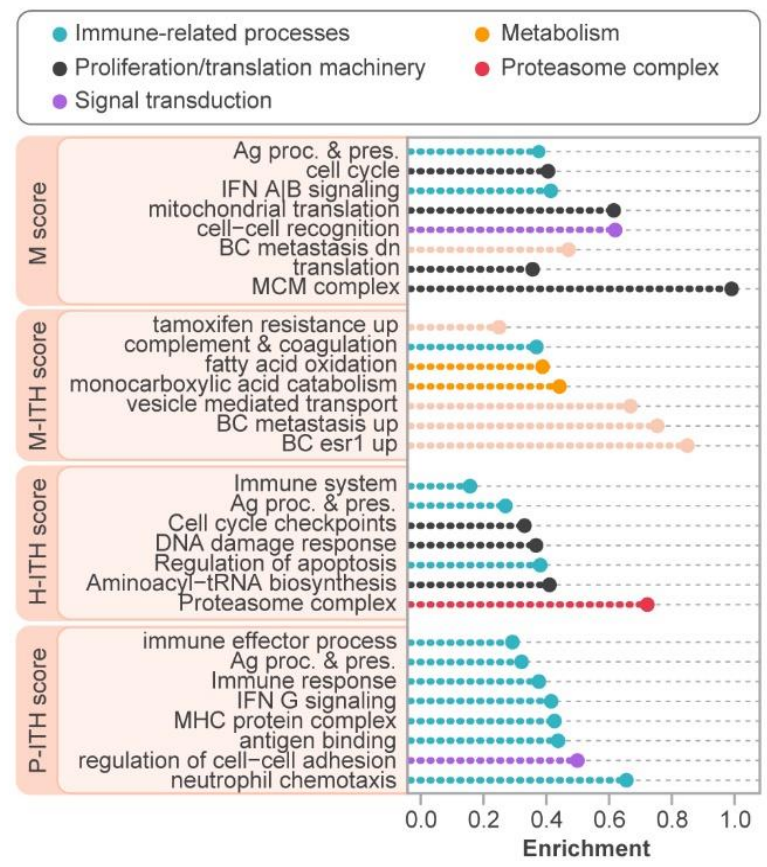

D

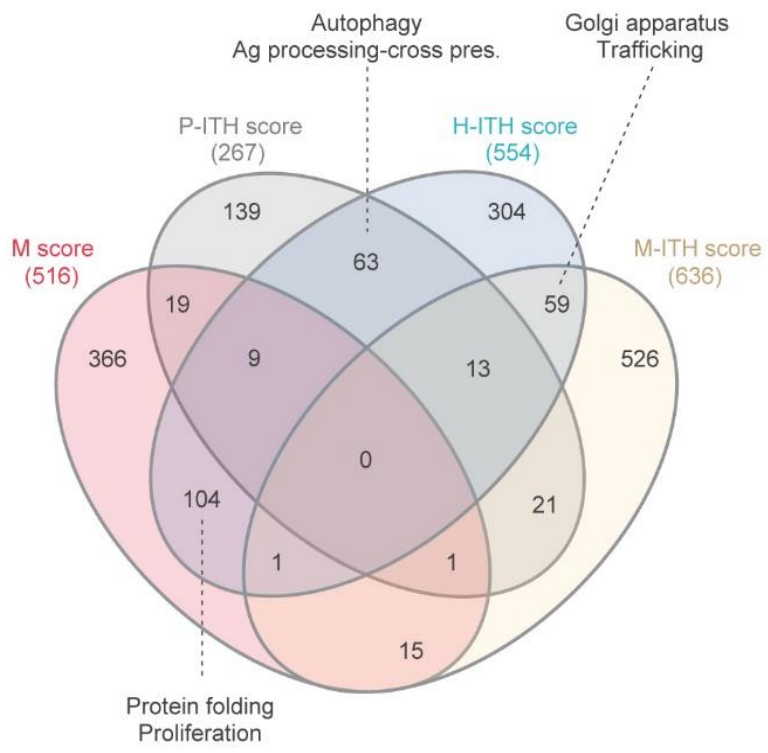

E

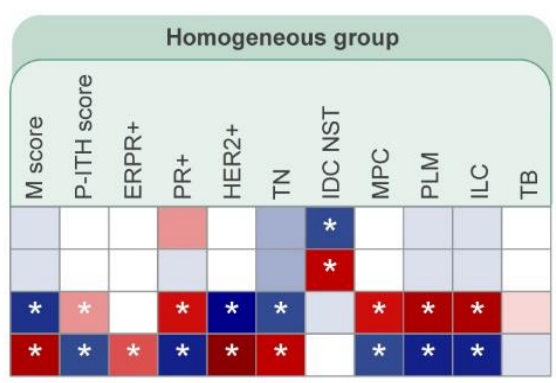

Non-invasive

Grade1

Grade2

Grade3

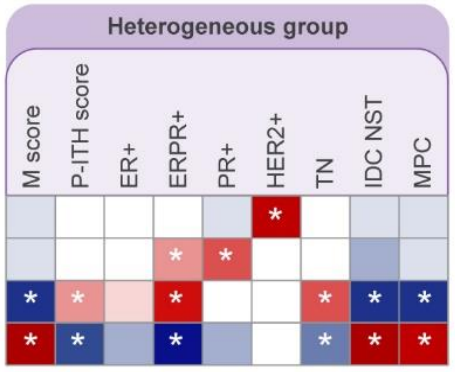

Spearman correlation

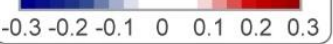


626 (A) 3D scatter plots of the first three components of the canonical correlation analysis of the 309 tumor regions data 627 were segregated based on their histological and molecular heterogeneity (top scatterplot) and tumor grade (bottom 628 scatterplot).

629 (B) Barplot shows the separation of the different tumor grades in the groups of histological and molecular 630 homogeneous and heterogeneous tumors $* * * p<0.00001$.

631 (C) Cleveland plot shows the functional enrichments of four scores (Benjamini-Hochberg FDR q-value < 0.02). The 632 entire list of enriched processes can be found in the Supplementary Table 3B.

633 (D) A Venn diagram shows the overlap of positively correlating proteins with each one of the scores.

634 (E) Correlation heatmap of clinical parameters separated based on the heterogeneity status of molecular and 635 histological subtypes. On the left heatmap, homogeneous tumors and on the right heatmap heterogeneous tumors. 636 Asterisk represents $p<0.05$ 
bioRxiv preprint doi: https://doi.org/10.1101/2021.08.05.455361; this version posted Auqust 6, 2021. The copyright holder for this preprint (which was not certified by peer review) is the author/funder, who has granted bioRxiv a license to display the preprint in perpetuity. It is made available under aCC-BY-NC 4.0 International license.

Mardamshina et al., Extended Data Fig. 5

A

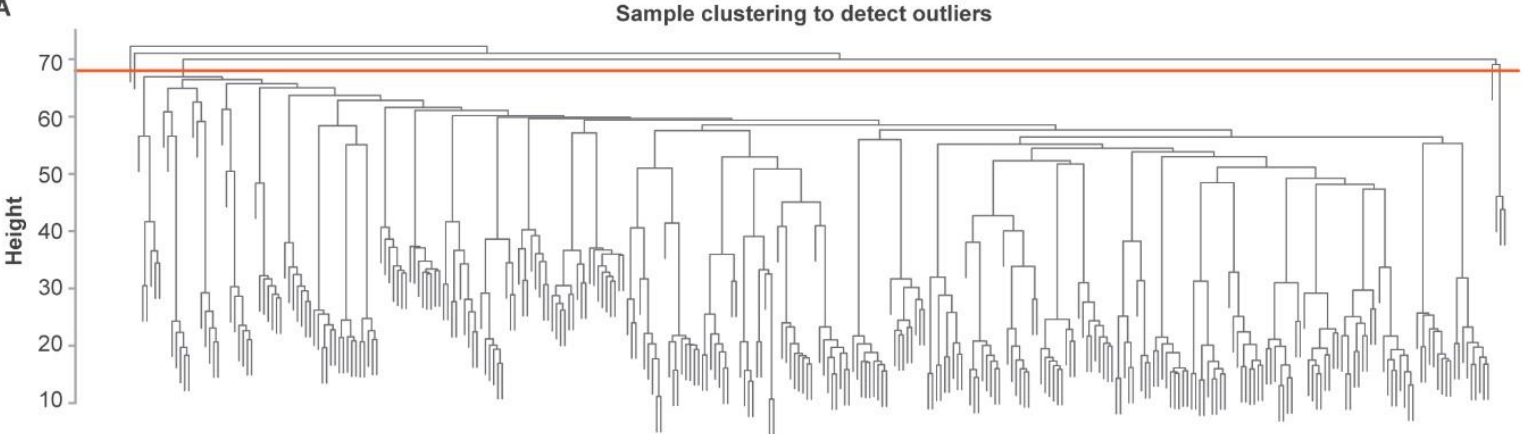

B
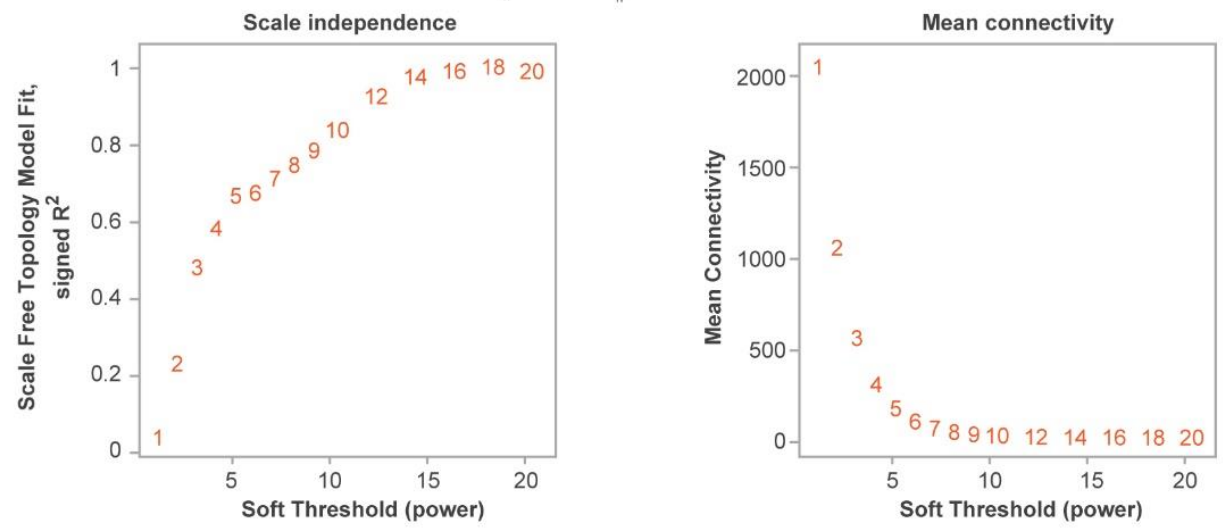

C

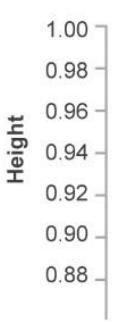

Module colors

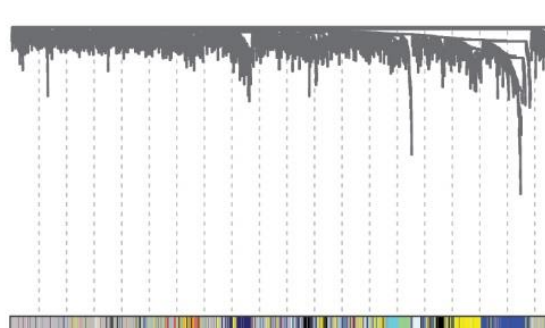

Cluster Dendrogram

D
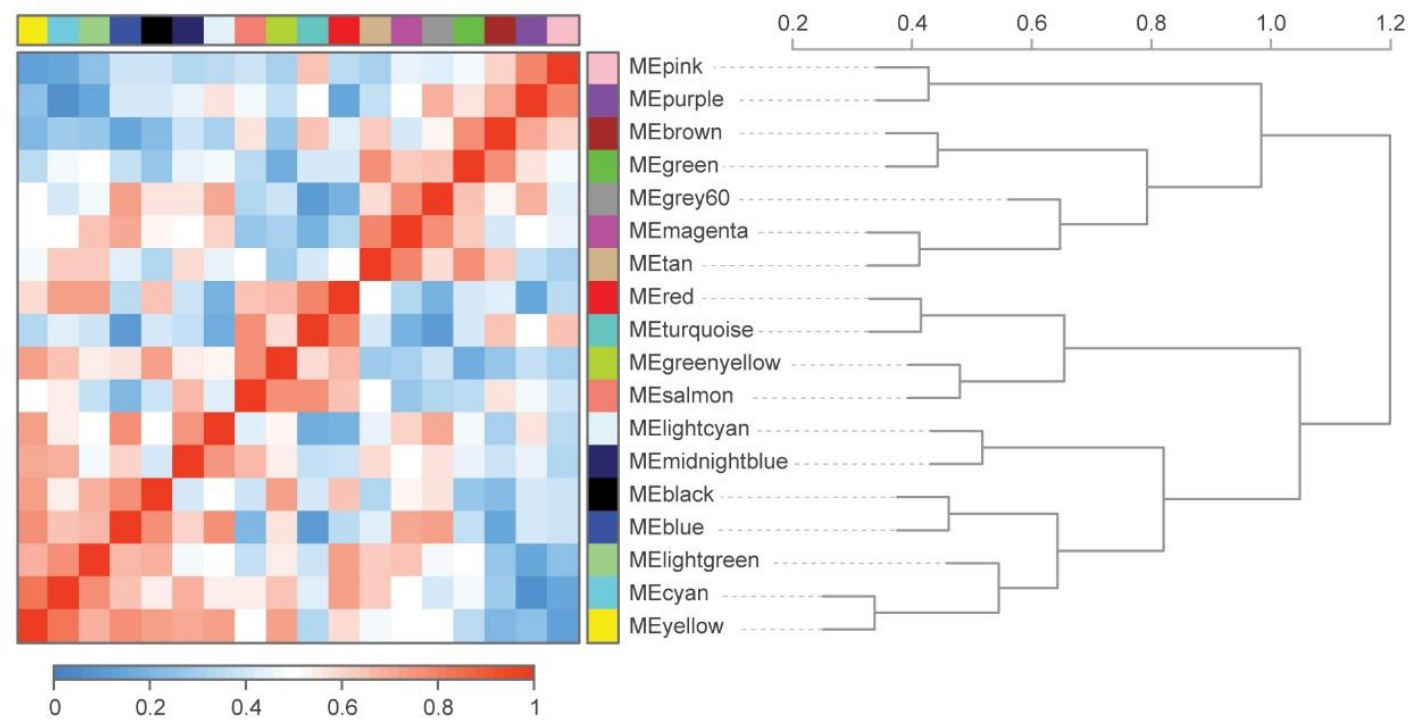


\section{Extended Data Fig. 5: WGCNA analysis.}

640 (A) Clustering of 330 regions within the WGCNA to remove outliers (red line). Six outlier samples were removed. 641 Three additional samples were removed due to missing annotations.

642 (B) Summary network indices ( $y$-axes) as functions of the soft thresholding power ( $x$-axes), where the numbers in 643 the plots indicate the corresponding soft thresholding powers. The plots indicate that approximate scale-free 644 topology is attained around the soft-thresholding power of 10.

645 (C) Protein dendrogram obtained by clustering the dissimilarity based on consensus Topological Overlap with the 646 corresponding module colors indicated by the color row. Consensus eigengene networks and its differential analysis 647 shown by clustering tree of the consensus module eigengenes and heatmap with high adjacency and low adjacency 648 to each module. 
bioRxiv preprint doi: https://doi.org/10.1101/2021.08.05.455361; this version posted August 6, 2021. The copyright holder for this preprint (which was not certified by peer review) is the author/funder, who has granted bioRxiv a license to display the preprint in perpetuity. It is made available under aCC-BY-NC 4.0 International license.

Mardamshina et al., Extended Data Fig. 6

A

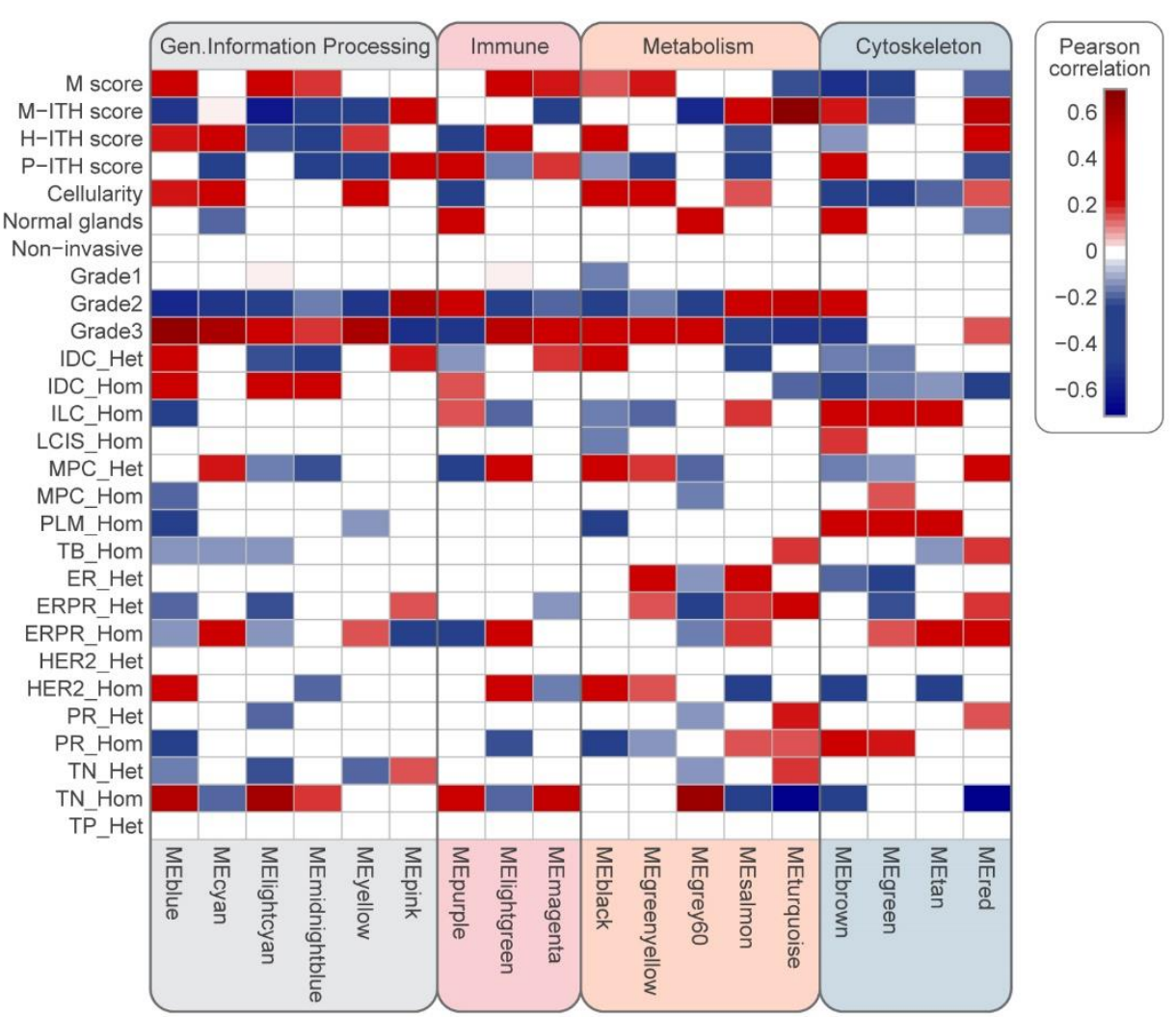

B

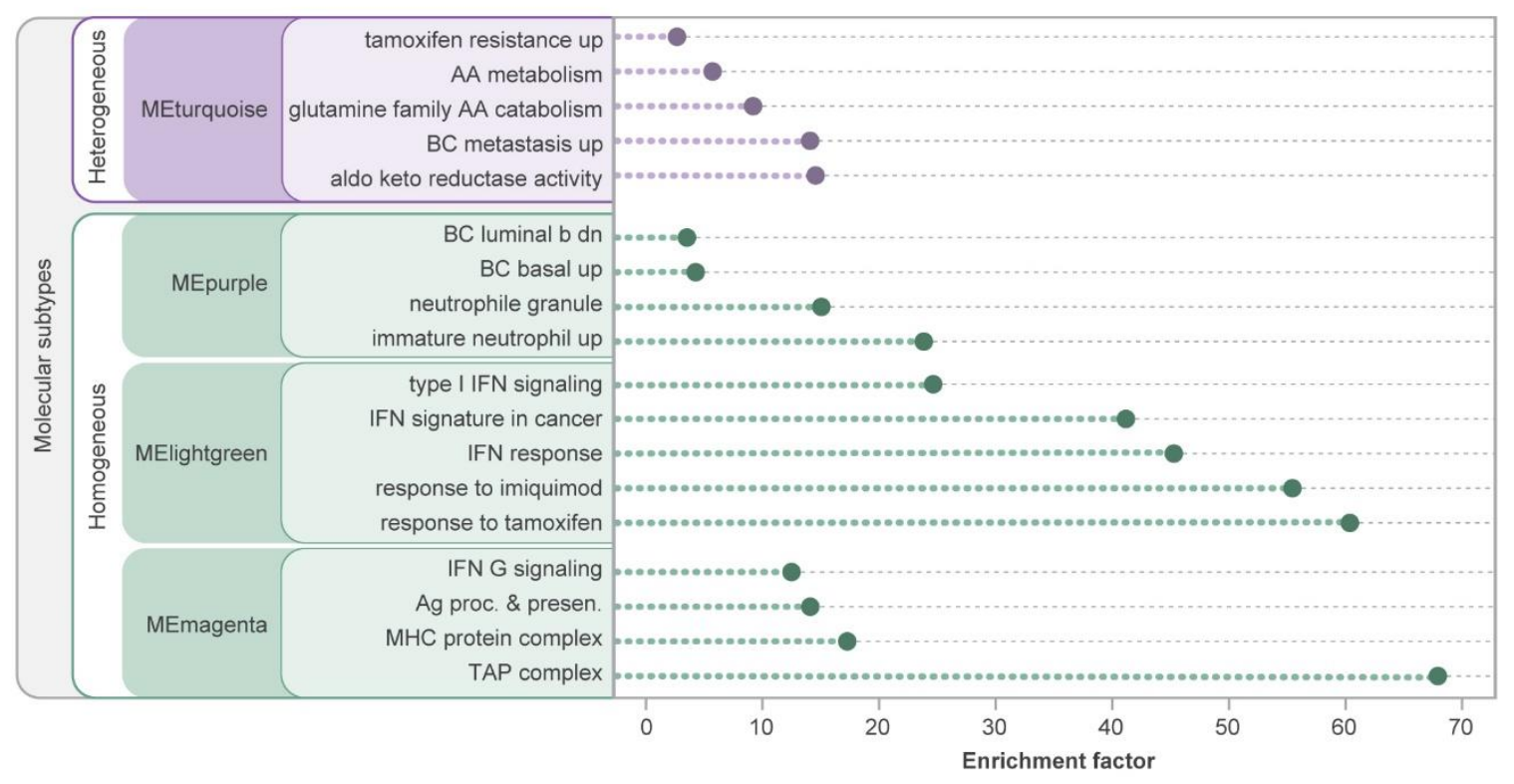

Extended Data Fig. 6: WGCNA of region proteomic data.

(A) WGCNA of 321 regions (tumor and normal tissue) shows module eigengenes (MEs) significantly correlating with different levels of heterogeneity and tumor grade (extended heatmap, related to Fig. 4). Only significant correlations are indicated (Pearson correlation, FDR q-value $<0.05$ ). 
bioRxiv preprint doi: https://doi.org/10.1101/2021.08.05.455361; this version posted August 6, 2021. The copyright holder for this preprint (which was not certified by peer review) is the author/funder, who has granted bioRxiv a license to display the preprint in perpetuity. It is made available under aCC-BY-NC 4.0 International license.

655

(B) Cleveland plot shows the functional enrichment of selected modules (FDR q-value $<0.02$ ). The entire list of enriched processes is given in the Supplementary Table 3C. 
bioRxiv preprint doi: https://doi.org/10.1101/2021.08.05.455361; this version posted August 6, 2021. The copyright holder for this preprint (which was not certified by peer review) is the author/funder, who has granted bioRxiv a license to display the preprint in perpetuity. It is made available under aCC-BY-NC 4.0 International license.

Mardamshina et al., Extended Data Fig. 7

A

IDC-NST

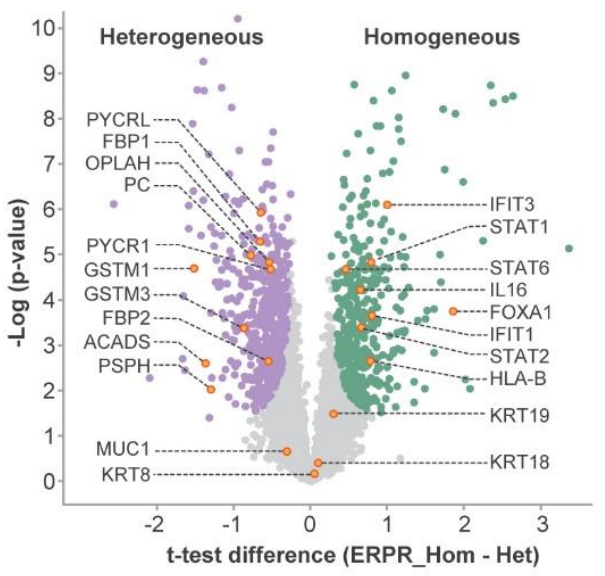

B

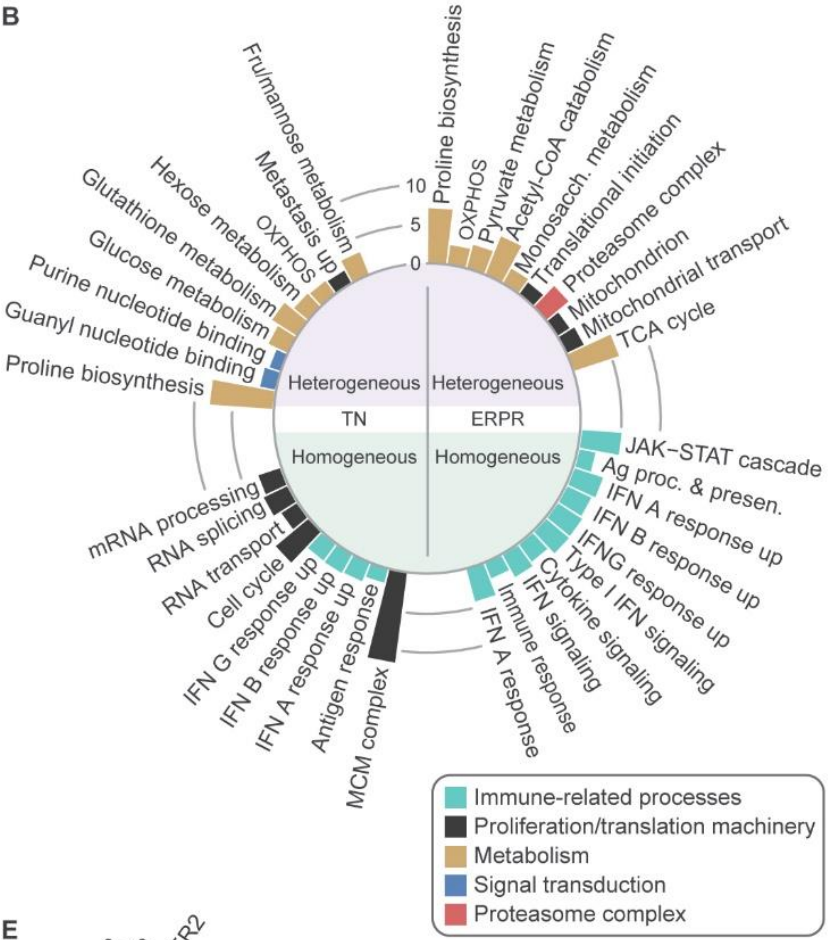

C

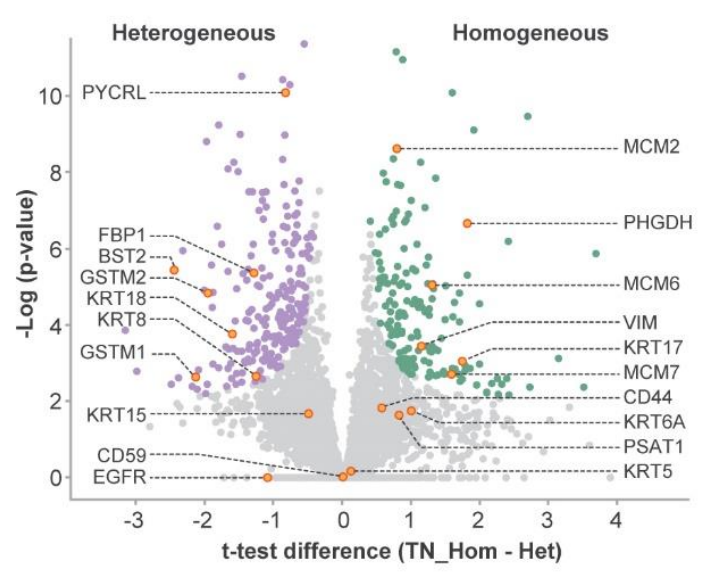

D

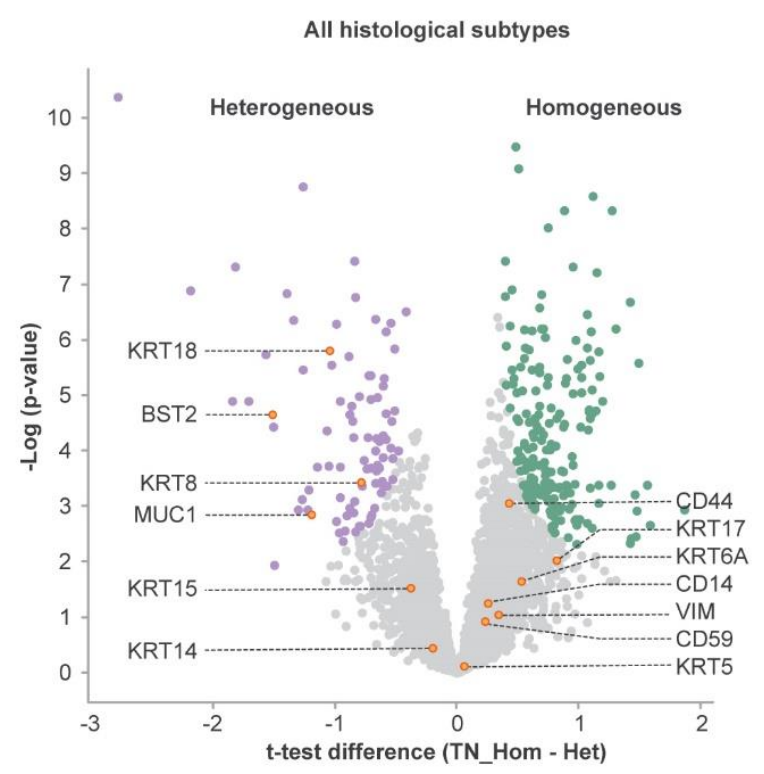

All histological subtypes

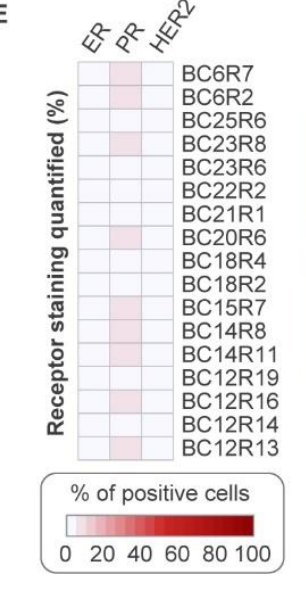

BC21

ER

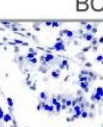
$200 \mathrm{~m}_{2} \mathrm{c} \cdot \mathrm{x}^{2} \mathrm{~s}$

PR

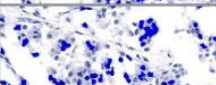

HER2

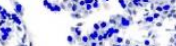

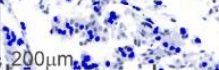

$\mathrm{BC} 20$

$\mathrm{BC} 22$

BC6

\begin{tabular}{l} 
PHGDH \\
MCM6 \\
VIM \\
KRT17 \\
MCM7 \\
CD44 \\
KRT6A \\
PSAT1 \\
KRT5 \\
\hline
\end{tabular}

IDC-NST 
658 Extended Data Fig. 7: Functional differences between heterogeneous and homogeneous regions.

659 (A) Volcano plots demonstrate the results of Welch's test comparing homogeneous and heterogeneous groups 660 based on molecular heterogeneity for ERPR+. The comparison was performed between 20 regions derived from five 661 patients (heterogeneous group) and 12 regions derived from two patients (homogeneous group).

662 (B) Enrichment analysis of significantly changing proteins from (A) and (B) (Fisher exact test Benjamini-Hochberg FDR 663 q-value < 0.02). Circular bar plot presents the enrichment factor. The entire list of enriched processes can be found 664 in Supplementary Table 3D.

665 (C) Volcano plot shows the results of Welch's test comparing homogeneous and heterogeneous TN regions. Markers 666 of basal subtype indicated below the cutoff threshold (grey); biomarkers of luminal subtype indicated in the 667 heterogeneous group (above the cutoff threshold). The comparison was performed between five regions from two 668 patients (heterogeneous group) and 36 regions from five patients (homogeneous group).

669 (D) Volcano plot shows the results of Welch's test comparing homogeneous and heterogeneous TN regions. The 670 comparison is similar to the one shown in (C), except the regions of grade 2 are represented by all the histological 671 subtypes across the cohort. The comparison was performed between 17 regions from nine patients (heterogeneous 672 group) and 37 regions from five patients (homogeneous group).

673 (E) Percentages of cells stained positive for ER, PR and HER2 and their combinations in the panel of selected 17 TN 674 regions from heterogeneous group that were used in (D). Examples of receptor staining per histological subtypes 675 from heterogeneous group of TN regions. 